\title{
SOME NEW INFINITE FAMILIES OF $\eta$-FUNCTION IDENTITIES
}

\author{
Verne E. Leininger and Stephen C. Milne \\ Dedicated to Professor Richard Askey on the occasion of his 65th birthday.
}

\begin{abstract}
In this paper, we utilize multiple basic hypergeometric series techniques to derive two new infinite families of $\eta$-function expansions, three infinite families of expansions of products of the form $\left(q^{a} ; q^{b}\right)_{\infty}$, and a $U(n+2)$ extension of the quintuple product identity. The $U(3)$ case of this extension immediately yields an elegant proof of the quintuple product identity. More specifically, we find expansions for $(q ; q)_{\infty}^{n^{2}+2}, \eta(q)^{n^{2}+2},(q ; q)_{\infty}^{n^{2}}, \eta(q)^{n^{2}},\left(q^{m} ; q^{m}\right)_{\infty}^{n^{2}}\left(q^{m-l}, q^{l} ; q^{m}\right)_{\infty}^{n}$ $\left(q^{l}, q^{m-l} ; q^{m}\right)_{\infty}\left(q^{m} ; q^{m}\right)_{\infty}^{n^{2}}$, and $\left(q^{m-l} ; q^{m}\right)_{\infty}\left(q^{m} ; q^{m}\right)_{\infty}^{n^{2}-1}$. All of these expansions, as well as the proof of the quintuple product identity, are consequences of various summation and transformation formulas for $U(n+1)$, equivalently $A_{n}$, multiple basic hypergeometric series. When we compare the powers of $\eta$ in our expansions with those in Macdonald's (1972) $\eta$-function expansions corresponding to affine root systems of type $B_{\ell}, C_{\ell}, B C_{\ell}$, and $D_{\ell}$, we utilize Chebyshev polynomials to show that the dimensions which give the same powers of $\eta$ consists of very sparse "Lucas sequences", which include the Pell numbers and the squares of the entries of the classical sequence which is the hypotenuse of the $n$-th Pythagorean triangle with consecutive integral legs.
\end{abstract}

\section{Introduction}

Ever since Euler proved that

$$
\prod_{i=1}^{\infty}\left(1-q^{i}\right)=\sum_{y=-\infty}^{\infty}(-1)^{y} q^{y(3 y+1) / 2}
$$

mathematicians have been looking for other identities of this form. In 1829, Jacobi $[12$, Section 66$]$ utilized his triple product identity to derive

$$
\prod_{i=1}^{\infty}\left(1-q^{i}\right)^{3}=\sum_{y=0}^{\infty}(-1)^{y}(2 y+1) q^{\left(y^{2}+y\right) / 2} .
$$

Since then, expansions have been found for $(q ; q)_{\infty}^{c}$ for many values of $c$ where

$$
0<|q|<1 \quad \text { and } \quad(\alpha)_{\infty} \equiv(\alpha ; q)_{\infty}:=\prod_{i=0}^{\infty}\left(1-\alpha q^{i}\right) .
$$

Received November 9, 1998.

1991 Mathematics Subject Classification: Primary 33D70, 05A19; Secondary 05A30, 05E99.

Key words and phrases: multiple basic hypergeometric series, very-well-poised on unitary groups $U(n+1)$, quintuple product identity, $\eta$-functions, Macdonald identities, Chebyshev polynomials, Pell's equation, Pell numbers, Pythagorean triangles, Lucas sequences, identities of Rogers-Ramanujan type. 
These included several infinite families of expansions and a few exceptional cases. See the introduction to [15] for more details.

In a 1972 paper, Macdonald [18] related most of these expansions for $(q ; q)_{\infty}^{c}$ to affine root systems. A few notable exceptions are: $c=2$ found by Hecke and Rogers, $c=4$ by Ramanujan, and $c=26$ by Atkin. In this paper, we add infinitely many more values to this list of exceptions by deriving expansions for the cases $c=n^{2}+2$ and $c=n^{2}$, and conjecturing one for $c=n^{2}-2$. We convert the cases $c=n^{2}+2$ and $c=n^{2}$ into expansions of these powers of the $\eta$-function by means of the definition

$$
\eta(q):=q^{1 / 24}(q ; q)_{\infty} .
$$

In Section 2, we utilize the multiple basic hypergeometric series techniques from [15] to derive the expansions for $\eta(q)^{n^{2}+2}$, and in Section 3, we obtain the expansions for $\eta(q)^{n^{2}}$. In [15], we derived new expansions for $(q ; q)_{\infty}^{n^{2}+2 n}$ and showed that they were equivalent to Macdonald's [18] $A_{n}$ family of expansions for $\eta(q)^{n^{2}+2 n}$. All of this work is motivated by the multiple basic hypergeometric series treatment of the Macdonald identities for $A_{\ell}^{(1)}$ in [19].

When we compare the powers of $\eta$ in our expansions in this paper with those in Macdonald's [18] $\eta$-function expansions corresponding to affine root systems of type $B_{\ell}, C_{\ell}, B C_{\ell}$, and $D_{\ell}$, we utilize Chebyshev polynomials to show that the dimensions which give the same powers of $\eta$ consist of very sparse "Lucas sequences" [27, pp. 5374], which include the Pell numbers [27, pp. 55 and 72] and the squares of the entries of the classical sequence [28, pp. 16-20] which is the hypotenuse of the $n$-th Pythagorean triangle with consecutive integral legs. We discuss this situation in greater detail in Section 6.

Even when the power of $\eta$ is the same, the sum sides of our identities and those of Macdonald [18] are generally in different dimensions-with ours being larger. However, our identities appear to be somewhat simpler. Other than one dimension, the identities are still different even in the rare case when the powers and dimensions are the same. Thus, our infinite families of $\eta$-function expansions appear to be new.

For convenience of illustration, consider the case $n=2$ of our expansion for $(q ; q)_{\infty}^{n^{2}+2}$ (see Theorem 2.2 below) given by

$$
(q ; q)_{\infty}^{6}=\sum_{-\infty<y_{1}, y_{2}<\infty}\left(y_{1}+y_{2}\right)\left[1+2\left(y_{2}-y_{1}\right)\right] q^{y_{1}^{2}+y_{2}^{2}+y_{2}}
$$

where $0<|q|<1$. Keeping in mind the lines of symmetry $y_{1}=0$ and $y_{2}=-1 / 2$, we rewrite (1.5) as

$$
(q ; q)_{\infty}^{6}=\sum_{y_{2}=0}^{\infty}\left(1+2 y_{2}\right)^{2} q^{y_{2}^{2}+y_{2}}+2 \sum_{y_{1}>0, y_{2} \geq 0}\left[1+4\left(y_{2}+y_{2}^{2}-y_{1}^{2}\right)\right] q^{y_{1}^{2}+y_{2}^{2}+y_{2}}
$$

where $0<|q|<1$. The $\eta$-function versions of (1.5) and (1.6) are not the same as the expansions for $\eta(q)^{6}$ in Macdonald's $B C_{2}$ identity [18, pp. 137-138, (6)(c)], or Ramanujan's identity [24, p. 176 (Sec. 19), and pp. 178-179 (Sec. 22)].

Macdonald [18] gave a variety of identities involving products and quotients of $\eta$ functions. More recently, similar identities for $\eta$-functions were found by several other authors including [4], [14], [16], and [32]. In Section 4 of this paper, we give expansions 
for

$$
\begin{gathered}
\left(q^{m} ; q^{m}\right)_{\infty}^{n^{2}}\left(q^{m-l}, q^{l} ; q^{m}\right)_{\infty}^{n}, \quad\left(q^{l}, q^{m-l} ; q^{m}\right)_{\infty}\left(q^{m} ; q^{m}\right)_{\infty}^{n^{2}} \\
\text { and } \quad\left(q^{m-l} ; q^{m}\right)_{\infty}\left(q^{m} ; q^{m}\right)_{\infty}^{n^{2}-1}
\end{gathered}
$$

where, for products of $q$-shifted factorials, we use the more compact notation

$$
\left(a_{1}, a_{2}, \ldots, a_{m} ; q\right)_{\infty}=\left(a_{1} ; q\right)_{\infty}\left(a_{2} ; q\right)_{\infty} \cdots\left(a_{m} ; q\right)_{\infty} .
$$

While the products in (1.7) cannot be written as products or quotients of $\eta$-functions, they are still of the same general form as those cited above.

Another identity which has attracted much attention in the theory and application of $q$-series is the quintuple product identity in [8, Ex. 5.6, p. 134], [2, pp. 80-83], and [3]. (See (5.1) below.) In Section 5 of this paper, we give a new proof, by first deriving a multivariable $U(n+2)$ extension, of the quintuple product identity, and then applying the Jacobi triple product identity $[8,(1.6 .1)$, p. 12$]$ to the $n=1$, or $U(3)$ case. That is, the quintuple product identity is a natural consequence of a twodimensional $U(3)$ triple product identity and the classical $U(2)$ Jacobi triple product identity.

All of the expansions in this paper, as well as the proof of the quintuple product identity, are consequences of various summation and transformation formulas for $U(n+$ 1 ), equivalently $A_{n}$, multiple basic hypergeometric series. See [21] for more background on these series.

In addition to (1.3) and (1.8), the following notation is used throughout the remainder of this paper:

$$
(\alpha)_{m} \equiv(\alpha ; q)_{m}:=\prod_{i=0}^{m-1}\left(1-\alpha q^{i}\right)
$$

where $m$ is a nonnegative integer and $|q|<1$.

As motivation, note that $(\alpha ; q)_{m}=(\alpha ; q)_{\infty} /\left(\alpha q^{m} ; q\right)_{\infty}$ where $(\alpha ; q)_{\infty}$ is defined by (1.3).

\section{An expansion for $(q ; q)_{\infty}^{n^{2}+2}$}

In this section, we derive new expansions for $(q ; q)_{\infty}^{n^{2}+2}$ and $\eta(q)^{n^{2}+2}$.

We begin by setting $x=-t^{-1}$ and $z_{i}=x_{i}$ for $i=1, \ldots, n$ in Theorem 1.53 from [19]. This identity also can be derived by bilateralizing Theorem 5.44 from [21],

$$
\begin{aligned}
(t)_{\infty}\left(t^{-1} q\right)_{\infty}(q)_{\infty}^{n} \prod_{1 \leq r<s \leq n}\left[\left(\frac{x_{r}}{x_{s}}\right)_{\infty}\left(q \frac{x_{s}}{x_{r}}\right)_{\infty}\right] \\
=\sum_{\substack{-\infty<y_{i}<\infty \\
i=1, \ldots, n}}\left\{\prod_{1 \leq r<s \leq n}\left[1-\frac{x_{r}}{x_{s}} q^{y_{r}-y_{s}}\right] \prod_{i=1}^{n}\left[x_{i}^{n y_{i}-\left(y_{1}+\cdots+y_{n}\right)}\right]\right. \\
\quad \times(-1)^{n\left(y_{1}+\cdots+y_{n}\right)} q^{\left.n\left[\left(\begin{array}{c}
y_{1} \\
2
\end{array}\right)+\cdots+\left(\begin{array}{c}
y_{n} \\
2
\end{array}\right)\right]^{y_{1}+2 y_{2}+\cdots+n y_{n}}\right\} t^{-\left(y_{1}+\cdots+y_{n}\right)} .}
\end{aligned}
$$


We then apply the identity

$$
\prod_{1 \leq r<s \leq n}\left[1-\frac{x_{r}}{x_{s}} q^{y_{r}-y_{s}}\right]=\sum_{\sigma \in \mathcal{S}_{n}} \operatorname{sign}(\sigma) \prod_{i=1}^{n}\left[x_{i}^{\sigma(i)-i} q^{y_{i}(\sigma(i)-i)}\right]
$$

where $\mathcal{S}_{n}$ is the symmetric group on $n$ elements. Next, rearrange and permute the subscripts of $y$. This yields the identity:

$$
\begin{aligned}
&(1-t)(t q)_{\infty}\left(t^{-1} q\right)_{\infty}(q)_{\infty}^{n} \prod_{1 \leq r<s \leq n} {\left[\left(1-\frac{x_{r}}{x_{s}}\right)\left(q \frac{x_{r}}{x_{s}}\right)_{\infty}\left(q \frac{x_{s}}{x_{r}}\right)_{\infty}\right] } \\
&=\sum_{\substack{-\infty<y_{i}<\infty \\
i=1, \ldots, n}} \sum_{\sigma \in \mathcal{S}_{n}} \operatorname{sign}(\sigma)\left\{\prod_{i=1}^{n}\left[x_{i}^{n y_{\sigma(i)}-\left(y_{1}+\cdots+y_{n}\right)+\sigma(i)-i}\right]\right. \\
&\left.\times(-1)^{n\left(y_{1}+\cdots+y_{n}\right)} q^{\sum_{i=1}^{n}\left[\frac{n}{2} y_{i}^{2}+\left(i-\frac{n}{2}\right) y_{i}\right]}\right\} t^{-\left(y_{1}+\cdots+y_{n}\right)}
\end{aligned}
$$

where $n \geq 1$ and $0<|q|<1$.

Next, we take the derivative with respect to $t$ and set $t=1$. We also take $\partial\left(\begin{array}{c}n \\ 2\end{array}\right) / \partial x_{1}^{0} \partial x_{2}^{1} \cdots \partial x_{n}^{n-1}$ and then set $x_{1}=\cdots=x_{n}=1$. For more details on this process, see Section 3 of [15]. The motivation for using derivatives here is that they also simplify the standard proof of (1.2) in [11]. We have

$$
\begin{aligned}
& \prod_{i=1}^{n-1} i ! \cdot(q)_{\infty}^{n^{2}+2}=\sum_{\substack{-\infty<y_{i}<\infty \\
i=1, \ldots, n}} \sum_{\sigma \in \mathcal{S}_{n}} \operatorname{sign}(\sigma)\left\{(-1)^{n\left(y_{1}+\cdots+y_{n}\right)}\left(y_{1}+\cdots+y_{n}\right)\right. \\
& \left.\quad \times q^{\sum_{i=1}^{n}\left[\frac{n}{2} y_{i}^{2}+\left(i-\frac{n}{2}\right) y_{i}\right]} \prod_{i=1}^{n} \prod_{j=1}^{i-1}\left[n y_{\sigma(i)}-\left(y_{1}+\cdots+y_{n}\right)+\sigma(i)-i-(j-1)\right]\right\} .
\end{aligned}
$$

Observe that the inner sum in (2.4) depending on $\sigma \in \mathcal{S}_{n}$ can be rewritten as the determinant

$$
\operatorname{det}\left(c_{i j}\right)=\sum_{\sigma \in \mathcal{S}_{n}} \operatorname{sign}(\sigma) \prod_{i=1}^{n} c_{\sigma(i) i}
$$

where

$$
c_{i j}=\prod_{k=1}^{j-1}\left[n y_{i}-\left(y_{1}+\cdots+y_{n}\right)+i-j-(k-1)\right], \quad \text { if } j>1 \text { and } c_{i j}=1, \text { if } j=1 .
$$

We evaluate the determinant in (2.5) by a special case of the well-known generalized Vandermonde determinant formula in [31] and [23, Ex.2, p. 353 (see also pp. 340-341)]. The special case, also used in [15], that we need is 
Lemma 2.1 .

$$
\operatorname{det}\left|\begin{array}{ccccc}
1 & z_{1} & \left(z_{1}-1\right)\left(z_{1}-2\right) & \ldots & \left(z_{1}-n+2\right)\left(z_{1}-n+1\right) \cdots\left(z_{1}-2 n+4\right) \\
1 & z_{2} & \left(z_{2}-1\right)\left(z_{2}-2\right) & \ldots & \left(z_{2}-n+2\right)\left(z_{2}-n+1\right) \cdots\left(z_{2}-2 n+4\right) \\
\vdots & \vdots & \vdots & & \vdots \\
1 & z_{n} & \left(z_{n}-1\right)\left(z_{n}-2\right) & \ldots & \left(z_{n}-n+2\right)\left(z_{n}-n+1\right) \cdots\left(z_{n}-2 n+4\right)
\end{array}\right|
$$

Applying Lemma 2.1 to the determinant in (2.5) gives

$$
\operatorname{det}\left(c_{i j}\right)=\prod_{1 \leq r<s \leq n}\left[n y_{s}-n y_{r}+s-r\right] .
$$

Thus, we have proved the theorem:

Theorem 2.2. Let $n \geq 1$ and $|q|<1$. Then

$$
\begin{aligned}
(q ; q)_{\infty}^{n^{2}+2}= & \prod_{i=1}^{n-1} i !^{-1} \sum_{\substack{-\infty<y_{i}<\infty \\
y=1, \ldots, n}}\left\{(-1)^{n\left(y_{1}+\cdots+y_{n}\right)}\left(y_{1}+\cdots+y_{n}\right)\right. \\
& \left.\times \prod_{1 \leq r<s \leq n}\left[n y_{s}-n y_{r}+s-r\right] q^{\sum_{i=1}^{n}\left[\frac{n}{2} y_{i}^{2}+\left(i-\frac{n}{2}\right) y_{i}\right]}\right\} .
\end{aligned}
$$

The case $n=1$ of Theorem 2.2 is equivalent to (1.2), once we combine the $y_{1}$ and $-\left(y_{1}+1\right)$ terms in the sum side of $(2.9)$ for $y_{1} \geq 0$. The case $n=2$ of Theorem 2.2 is discussed in (1.5) and (1.6). It should be possible to transform Theorem 2.2 into a generalization of (1.2) and (1.6) by appealing to the symmetry techniques in Sections 4 and 5 of [15].

To put Theorem 2.2 into the form of an $\eta$-function identity, we let $y_{i}=\left(v_{i}-i\right) / n$ for $i=1, \ldots, n$. Note that since the $y_{i}$ 's are integers, the $v_{i}$ 's must satisfy $v_{i} \equiv i$ $(\bmod n)$. Recalling (1.4), we must multiply each side of Theorem 2.2 by $q^{\left(n^{2}+2\right) / 24}$. Thus, we obtain the following theorem.

Theorem 2.3. Let $n \geq 1$ and $|q|<1$. Then

$$
\begin{aligned}
\eta(q)^{n^{2}+2}=(-1)^{\left(\begin{array}{c}
n+1 \\
2
\end{array}\right)} \prod_{i=1}^{n-1} i !^{-1} \sum_{\substack{v_{i} \equiv i \in \mathbb{Z}^{n} \\
i=1, \ldots, n}}\left\{\left[\frac{v_{1}+\cdots+v_{n}}{n}-\frac{n+1}{2}\right]\right. \\
\left.\times(-1)^{v_{1}+\cdots+v_{n}} \prod_{1 \leq r<s \leq n}\left[v_{s}-v_{r}\right] q^{\frac{1}{2 n} \sum_{i=1}^{n}\left(v_{i}-n / 2\right)^{2}}\right\} .
\end{aligned}
$$

\section{An expansion for $(q ; q)_{\infty}^{n^{2}}$}

In this section, we derive a new expansion for $(q ; q)_{\infty}^{n^{2}}$. This is accomplished by first using an identity of Euler and a diagonal sum argument to rewrite Theorem 1.58 of [19]. We then appeal to the differentiation techniques in Section 3 of [15] and the classical determinant evaluation in Lemma 2.1. 

by

We start with the case $z \mapsto-t q$ of an identity of Euler from [8, p. 9, (1.3.16)] given

$$
(t q ; q)_{\infty}=\sum_{m=0}^{\infty} \frac{(-1)^{m} q^{m(m+1) / 2} t^{m}}{(q ; q)_{m}}
$$

where $(q ; q)_{m}$ is determined by (1.9). Theorem 1.58 of [19] can be written as

$$
\begin{gathered}
(q)_{\infty}^{n-1} \prod_{\substack{1 \leq r<s \leq n \\
\times}}\left[\left(\frac{x_{r}}{x_{s}}\right)_{\infty}\left(q \frac{x_{s}}{x_{r}}\right)_{\infty}\right]=(-1)^{(n-1) m}\left(x_{1} \cdots x_{n}\right)^{-m} q^{-m(m+1) / 2} \\
\sum_{\substack{-\infty<y_{i}<\infty \\
y_{1}+\cdots+y_{n}=m}}\left\{\prod_{\substack{1 \leq r<s \leq n \\
x_{s}}}\left[1-\frac{x_{r}}{x_{s}} q^{y_{r}-y_{s}}\right]\right. \\
\left.\times \prod_{i=1}^{n}\left[x_{i}^{n y_{i}}\right] q^{n\left[\left(\begin{array}{c}
y_{1} \\
2
\end{array}\right)+\cdots+\left(\begin{array}{c}
y_{n} \\
2
\end{array}\right)\right]} q^{y_{1}+2 y_{2}+\cdots+n y_{n}}\right\}
\end{gathered}
$$

where $m$ is any integer. Now, multiplying both sides of (3.1) by the left side of (3.2), using the relation (3.2), interchanging summations, and then summing over the diagonals $\left\{y_{1}, \ldots, y_{n} \geq 0\right.$ such that $\left.y_{1}+\cdots+y_{n}=m\right\}$ from $m=0$ to $m=\infty$ immediately gives

$$
\begin{aligned}
(t q)_{\infty}(q)_{\infty}^{n-1} \prod_{1 \leq r<s \leq n}\left[\left(\frac{x_{r}}{x_{s}}\right)_{\infty}\left(q \frac{x_{s}}{x_{r}}\right)_{\infty}\right] \\
=\sum_{\substack{-\infty<y_{i}<\infty \\
y_{1}+\cdots+y_{n} \geq 0}}\left\{\prod_{1 \leq r<s \leq n}\left[1-\frac{x_{r}}{x_{s}} q^{y_{r}-y_{s}}\right] \prod_{i=1}^{n}\left[x_{i}^{n y_{i}-\left(y_{1}+\cdots+y_{n}\right)}\right]\right. \\
\left.\quad \times(q)_{y_{1}+\cdots+y_{n}}^{-1}(-1)^{n\left(y_{1}+\cdots+y_{n}\right)} q^{n\left[\left(\begin{array}{c}
y_{1} \\
2
\end{array}\right)+\cdots+\left(\begin{array}{c}
y_{n} \\
2
\end{array}\right)\right]} q^{y_{1}+2 y_{2}+\cdots+n y_{n}}\right\} t^{y_{1}+\cdots+y_{n}}
\end{aligned}
$$

where $n \geq 1$ and $0<|q|<1$.

The identity in (3.3) can also be derived by bilateralizing Theorem 5.50 from [21] "over a triangle". We first shift the index of summation for each $y_{i}$ down by $N / n$, shift the $y_{i}$ 's up by $N / n$, simplify, then replace $y_{i}, z$, and $x_{i}$ by $-y_{i}, z^{-1}$, and $x_{i}^{-1}$, respectively, and finally take $N \mapsto m n$ and let $m \mapsto \infty$. Some elementary simplification of the product side then yields (3.3).

We next apply the identity (2.2) to (3.3), rearrange, and then permute the subscripts of $y$. This yields the identity:

$$
\begin{aligned}
& (t q)_{\infty}(q)_{\infty}^{n-1} \prod_{\substack{1 \leq r<s \leq n\\
}}\left[\left(1-\frac{x_{r}}{x_{s}}\right)\left(q \frac{x_{r}}{x_{s}}\right)_{\infty}\left(q \frac{x_{s}}{x_{r}}\right)_{\infty}\right] \\
& =\sum_{\substack{-\infty<y_{i}<\infty \\
y_{1}+\cdots+y_{n} \geq 0}} \sum_{\sigma \in \mathcal{S}_{n}} \operatorname{sign}(\sigma)\left\{\prod_{i=1}^{n}\left[x_{i}^{n y_{\sigma(i)}-\left(y_{1}+\cdots+y_{n}\right)+\sigma(i)-i}\right]\right. \\
& \left.\quad \times(q)_{y_{1}+\cdots+y_{n}}^{-1}(-1)^{n\left(y_{1}+\cdots+y_{n}\right)} q^{\sum_{i=1}^{n}\left[\frac{n}{2} y_{i}^{2}+\left(i-\frac{n}{2}\right) y_{i}\right]}\right\} t^{y_{1}+\cdots+y_{n}} .
\end{aligned}
$$


Set $t=1$. We also take $\partial^{\left(\begin{array}{c}n \\ 2\end{array}\right)} / \partial x_{1}^{0} \partial x_{2}^{1} \cdots \partial x_{n}^{n-1}$ and then set $x_{1}=\cdots=x_{n}=1$. For more details on this process, see Section 3 of [15]. We have

$$
\begin{aligned}
\prod_{i=1}^{n-1} i ! \cdot(q)_{\infty}^{n^{2}}= & \sum_{\substack{-\infty<y_{i}<\infty \\
y_{1}+\cdots+y_{n} \geq 0}} \sum_{\sigma \in \mathcal{S}_{n}} \operatorname{sign}(\sigma)\left\{(-1)^{n\left(y_{1}+\cdots+y_{n}\right)}(q)_{y_{1}+\cdots+y_{n}}^{-1} q^{\sum_{i=1}^{n}\left[\frac{n}{2} y_{i}^{2}+\left(i-\frac{n}{2}\right) y_{i}\right]}\right. \\
& \left.\times \prod_{i=1}^{n} \prod_{j=1}^{i-1}\left[n y_{\sigma(i)}-\left(y_{1}+\cdots+y_{n}\right)+\sigma(i)-i-(j-1)\right]\right\}
\end{aligned}
$$

The same analysis as in (2.5)-(2.8) when applied to the inner sum in (3.5), which depends on $\sigma \in \mathcal{S}_{n}$, transforms (3.5) into the following theorem.

Theorem 3.1. Let $n \geq 1$ and $|q|<1$. Then

$$
\begin{aligned}
(q ; q)_{\infty}^{n^{2}}= & \prod_{i=1}^{n-1} i !^{-1} \sum_{\substack{-\infty<y_{i}<\infty \\
y_{1}+\cdots+y_{n} \geq 0}}\left\{(-1)^{n\left(y_{1}+\cdots+y_{n}\right)}(q ; q)_{y_{1}+\cdots+y_{n}}^{-1}\right. \\
& \left.\times \prod_{1 \leq r<s \leq n}\left[n y_{s}-n y_{r}+s-r\right] q^{\sum_{i=1}^{n}\left[\frac{n}{2} y_{i}^{2}+\left(i-\frac{n}{2}\right) y_{i}\right]}\right\} .
\end{aligned}
$$

The case $n=1$ of Theorem 3.1 is the case $t \mapsto 1$ of (3.1).

Just as for Theorem 2.3, multiplying each side of Theorem 3.1 by $q^{\left(n^{2}\right) / 24}$ gives the theorem:

Theorem 3.2. Let $n \geq 1$ and $|q|<1$. Then

$$
\begin{aligned}
\eta(q)^{n^{2}}=(-1)^{\left(\begin{array}{c}
n+1 \\
2
\end{array}\right)} q^{-1 / 12} \prod_{i=1}^{n-1} i !^{-1} \sum_{\substack{v_{i} \equiv i \\
\nu_{1}+\cdots+\nu_{n} \geq\left(\begin{array}{c}
n+1 \\
2
\end{array}\right)}}(-1)^{\nu_{1}+\cdots+\nu_{n}}(q)_{(1 / n)\left(\nu_{1}+\cdots+\nu_{n}-(n+1) n / 2\right)}^{-1} \\
\quad \times \prod_{1 \leq r<s \leq n}\left[\nu_{s}-\nu_{r}\right] q^{\left.(1 / 2 n) \sum_{i=1}^{n}\left(\nu_{i}-n / 2\right)^{2}\right]} .
\end{aligned}
$$

We conclude this section with a formal identity that yields a conjectured expansion for $(q ; q)_{\infty}^{n^{2}-2}$. We use the term "formal identity" since our application of the multiple power series ratio test did not establish absolute convergence of the multiple series involved here. We describe this situation in more detail following equation (3.9) below.

We begin with the case $z \mapsto t$ of another identity of Euler from [8, p. 9, (1.3.15)] given by

$$
(t ; q)_{\infty}^{-1}=\sum_{m=0}^{\infty} \frac{t^{m}}{(q ; q)_{m}}
$$


with $|t|<1$. An analysis just like that applied to (3.1) to (3.3) when applied to (3.8) immediately gives

$$
\begin{aligned}
(t)_{\infty}^{-1}(q)_{\infty}^{n-1} \prod_{1 \leq r<s \leq n} & {\left[\left(\frac{x_{r}}{x_{s}}\right)_{\infty}\left(q \frac{x_{s}}{x_{r}}\right)_{\infty}\right] } \\
= & \sum_{\substack{-\infty<y_{i}<\infty \\
y_{1}+\cdots+y_{n} \geq 0}}\left\{\prod_{1 \leq r<s \leq n}\left[1-\frac{x_{r}}{x_{s}} q^{y_{r}-y_{s}}\right] \prod_{i=1}^{n}\left[x_{i}^{n y_{i}-\left(y_{1}+\cdots+y_{n}\right)}\right]\right. \\
& \times(q)_{y_{1}+\cdots+y_{n}}^{-1}(-1)^{(n-1)\left(y_{1}+\cdots+y_{n}\right)} q^{-e_{2}\left(y_{1}, \cdots, y_{n}\right)} \\
& \left.\times q^{(n-1)\left[\left(\frac{y_{1}}{2}\right)+\cdots+\left(\frac{y_{n}}{2}\right)\right]} q^{y_{2}+2 y_{3}+\cdots+(n-1) y_{n}}\right\} t^{y_{1}+\cdots+y_{n}}
\end{aligned}
$$

where $0<|q|<1$, suitable convergence conditions hold, $e_{2}\left(y_{1}, \ldots, y_{n}\right)$ is the second elementary symmetric function of $\left\{y_{1}, \ldots, y_{n}\right\}$, and $n \geq 1$.

In studying the convergence of (3.9) and the other multiple series in this paper, we utilize the analysis in [21, p. 134] that depended on the following multiple power series ratio test.

Lemma 3.3. Let $f\left(y_{1}, \ldots, y_{n}\right)$ be a function of $\left\{y_{1}, \ldots, y_{n}\right\}$. Given the multiple series $\sum_{\left\{y_{1}, \ldots, y_{n}\right\} \geq 0} f\left(y_{1}, \ldots, y_{n}\right)$, set

$$
g_{m}\left(y_{1}, \ldots, y_{n}\right):=\left|\frac{f\left(y_{1}, \ldots, y_{m-1}, y_{m}+1, y_{m+1}, \ldots, y_{n}\right)}{f\left(y_{1}, \ldots, y_{n}\right)}\right|
$$

for $m=1, \ldots, n$. Then, if

$$
\lim _{\varepsilon \rightarrow \infty} g_{m}\left(\varepsilon y_{1}, \ldots, \varepsilon y_{n}\right)<1
$$

for $m=1, \ldots, n$, the multiple series converges absolutely.

Before applying Lemma 3.3 to check the convergence of (3.9), we utilize the comparison test and consider the dominating multiple series determined by replacing

$$
q^{(n-1)\left[\left(\begin{array}{c}
y_{1} \\
2
\end{array}\right)+\cdots+\left(\begin{array}{c}
y_{n} \\
2
\end{array}\right)\right]} q^{-e_{2}\left(y_{1}, \ldots, y_{n}\right)}
$$

by

$$
q^{-((n-1) / 2)\left(y_{1}+\cdots+y_{n}\right)}
$$

This step depends upon the identity

$$
\begin{aligned}
(n-1) & {\left[\left(\begin{array}{c}
y_{1} \\
2
\end{array}\right)+\cdots+\left(\begin{array}{c}
y_{n} \\
2
\end{array}\right)\right]-e_{2}\left(y_{1}, \ldots, y_{n}\right) } \\
= & -\frac{(n-1)}{2}\left(y_{1}+\cdots+y_{n}\right)+\frac{1}{2} \sum_{1 \leq r<s \leq n}\left(y_{r}-y_{s}\right)^{2} .
\end{aligned}
$$

Now, for the dominating multiple series, apply (2.2), interchange the summations, and then apply Lemma 3.3 to the multiple power series arising from each of the resulting $n$ ! inner multiple sums. We find that some inner multiple power series require at least $0<|q|<1$ in order to converge absolutely, while the rest need $1<|q|$. If one of these conditions is true, the other is false. Thus, this application of Lemma 3.3 to (3.9) is inconclusive. A more delicate analysis is required to establish the convergence of (3.9). On the other hand, a similar (or even simpler) analysis is sufficient to establish convergence in the rest of this paper. 
We next carry out an analysis similar to that in (3.4) to (3.6). Apply (2.2) to (3.9), rearrange, and permute the subscripts of $y$. In the resulting identity, set $t=q$, then take $\partial^{\left(\begin{array}{c}n \\ 2\end{array}\right)} / \partial x_{1}^{0} \partial x_{2}^{1} \cdots \partial x_{n}^{n-1}$, and finally set $x_{1}=\cdots=x_{n}=1$. Again, apply the classical determinant evaluation in Lemma 2.1. We obtain the conjecture:

Conjecture 3.4. Let $n \geq 1$ and $0<|q|<1$. Assume that suitable convergence conditions hold. Then

$$
\begin{aligned}
& (q ; q)_{\infty}^{n^{2}-2}=\prod_{i=1}^{n-1} i !^{-1} \sum_{\substack{-\infty<y_{i}<\infty \\
y_{1}+\cdots+y_{n} \geq 0}}\left\{(-1)^{(n-1)\left(y_{1}+\cdots+y_{n}\right)}(q ; q)_{y_{1}+\cdots+y_{n}}^{-1}\right. \\
& \left.\quad \times q^{-e_{2}\left(y_{1}, \ldots, y_{n}\right)} q^{\sum_{i=1}^{n}\left[\frac{(n-1)}{2} y_{i}^{2}+\left(i-\frac{(n-1)}{2}\right) y_{i}\right]} \prod_{\substack{1 \leq r<s \leq n \\
n^{2}}}\left[n y_{s}-n y_{r}+s-r\right]\right\}
\end{aligned}
$$

where $e_{2}\left(y_{1}, \ldots, y_{n}\right)$ is the second elementary symmetric function of $\left\{y_{1}, \ldots, y_{n}\right\}$.

The case $n=1$ of (3.15) is just the case $t=q$ of (3.8). The case $n=2$ is not the same as that found by Hecke and Rogers.

The case $n=2$ of Conjecture 3.4 is given by

$$
(q ; q)_{\infty}^{2}=\sum_{\substack{-\infty<y_{1}, y_{2}<\infty \\ y_{1}+y_{2} \geq 0}} \frac{\left[1+2\left(y_{2}-y_{1}\right)\right]}{(q ; q)_{y_{1}+y_{2}}}(-1)^{y_{1}+y_{2}} q^{\left[\left(y_{1}-y_{2}\right)^{2}+y_{1}+3 y_{2}\right] / 2}
$$

where $0<|q|<1$. We have checked with Mathematica [33] that both sides of (3.16) agree up to $q^{300}$.

The case $n=4$ of Conjecture 3.4 yields an expansion for $\eta(q)^{14}$ that is different from the $G_{2}$ expansion for $\eta(q)^{14}$ in [18, pp. 141-142, (6)].

\section{Some expansions for products of powers of $\left(q^{a} ; q^{m}\right)_{\infty}$}

In this section, we derive three infinite families of expansions for the products of powers of $\left(q^{a} ; q^{m}\right)_{\infty}$ which are listed in (1.7).

The first expansion is a consequence of the $U(n+1)$ generalization of the Jacobi triple product identity given by Theorem 1.2 from [22]. That is, we start with the theorem:

Theorem 4.1 (A $U(n+1)$ generalization of the Jacobi triple product identity). Let $t$ and $x_{1}, \ldots, x_{n}$ be indeterminate, and let $n \geq 1$. Suppose that none of the denominators in (4.1) vanishes, and that $0<|q|<1$. Then

$$
\begin{aligned}
& \sum_{-\infty<y_{1}, \ldots, y_{n}<\infty}\left\{\prod_{1 \leq r<s \leq n}\left[1-\frac{x_{r}}{x_{s}} q^{y_{r}-y_{s}}\right] \prod_{i=1}^{n}\left[\left(x_{i}\right)^{n y_{i}-\left(y_{1}+\cdots+y_{n}\right)}\right]\right. \\
& \times(-1)^{(n-1)\left(y_{1}+\cdots+y_{n}\right)} q^{\left[n\left[\left(\frac{y_{1}}{2}\right)+\cdots+\left(\begin{array}{c}
y_{n} \\
2
\end{array}\right)\right]+y_{1}+2 y_{2}+\cdots+n y_{n}\right]} \\
& \left.\times \prod_{i=1}^{n}\left[\left(\frac{x_{i}}{x_{n}}\right)^{y_{i}}\right] q^{-e_{2}\left(y_{1}, \ldots, y_{n}\right)}\right\} t^{y_{1}+\cdots+y_{n}} \\
= & \prod_{1 \leq r<s \leq n}\left[\left(\frac{x_{r}}{x_{s}}\right)_{\infty}\left(q \frac{x_{s}}{x_{r}}\right)_{\infty}\right] \prod_{i=1}^{n}\left[\left(-t q \frac{x_{i}}{x_{n}}\right)_{\infty}\left(-t^{-1} \frac{x_{n}}{x_{i}}\right)_{\infty}(q)_{\infty}\right]
\end{aligned}
$$

where $e_{2}\left(y_{1}, \ldots, y_{n}\right)$ is the second elementary symmetric function of $\left\{y_{1}, \ldots, y_{n}\right\}$. 
Apply identity (2.2) to (4.1), rearrange, and permute the subscripts of $y$. This yields the identity:

$$
\begin{aligned}
& \quad \sum_{-\infty<y_{1}, \ldots, y_{n}<\infty} \sum_{\sigma \in \mathcal{S}_{n}} \operatorname{sign}(\sigma)\left\{\prod_{i=1}^{n-1}\left[x_{i}^{(n+1) y_{\sigma(i)}-\left(y_{1}+\cdots+y_{n}\right)+\sigma(i)-i}\right]\right. \\
& \times x_{n}^{(n+1) y_{\sigma(n)}-2\left(y_{1}+\cdots+y_{n}\right)+\sigma(n)-n}(-1)^{(n-1)\left(y_{1}+\cdots+y_{n}\right)} \\
& \left.\times q^{\sum_{i=1}^{n}\left[(n / 2) y_{i}^{2}+(i-n / 2) y_{i}\right]-e_{2}\left(y_{1}, \ldots, y_{n}\right)}\right\} t^{y_{1}+\cdots+y_{n}} \\
& =\prod_{1 \leq r<s \leq n}\left[\left(\frac{x_{r}}{x_{s}}\right)_{\infty}\left(q \frac{x_{s}}{x_{r}}\right)_{\infty}\right] \prod_{i=1}^{n}\left[\left(-t q \frac{x_{i}}{x_{n}}\right)_{\infty}\left(-t^{-1} \frac{x_{n}}{x_{i}}\right)_{\infty}(q)_{\infty}\right] .
\end{aligned}
$$

Let $m \geq 2$ be an integer and let $l$ be an integer such that $1 \leq l<m$. Replace $q$ by $q^{m}$ and then replace $t$ by $-t q^{-l}$. We obtain

$$
\begin{aligned}
\sum_{-\infty<y_{1}, \ldots, y_{n}<\infty} & \sum_{\sigma \in \mathcal{S}_{n}} \operatorname{sign}(\sigma)\left\{\prod_{i=1}^{n-1}\left[x_{i}^{(n+1) y_{\sigma(i)}-\left(y_{1}+\cdots+y_{n}\right)+\sigma(i)-i}\right]\right. \\
\times & x_{n}^{(n+1) y_{\sigma(n)}-2\left(y_{1}+\cdots+y_{n}\right)+\sigma(n)-n}(-1)^{n\left(y_{1}+\cdots+y_{n}\right)} \\
& \left.\times q^{\sum_{i=1}^{n}\left[(m n / 2) y_{i}^{2}+(m i-m n / 2-l) y_{i}\right]-m e_{2}\left(y_{1}, \ldots, y_{n}\right)}\right\} t^{y_{1}+\cdots+y_{n}} \\
= & \prod_{1 \leq r<s \leq n}\left[\left(\frac{x_{r}}{x_{s}}, q^{m} \frac{x_{s}}{x_{r}} ; q^{m}\right)_{\infty}\right] \prod_{i=1}^{n}\left[\left(t q^{m-l} \frac{x_{i}}{x_{n}}, t^{-1} q^{l} \frac{x_{n}}{x_{i}}, q^{m} ; q^{m}\right)_{\infty}\right]
\end{aligned}
$$

where we have used the standard notation for products of $q$-shifted factorials in (1.8).

Set $t=1$ and $x_{n}=1$. Take $\partial_{\left(\begin{array}{c}n \\ 2\end{array}\right)} / \partial x_{1} \partial x_{2}^{2} \ldots \partial x_{n-1}^{n-1}$ and then set $x_{1}=\cdots=x_{n-1}$ $=1$. We have

$$
\begin{array}{r}
\sum_{-\infty<y_{1}, \ldots, y_{n}<\infty} \sum_{\sigma \in \mathcal{S}_{n}} \operatorname{sign}(\sigma)\left\{\prod _ { i = 1 } ^ { n - 1 } \prod _ { j = 1 } ^ { i } \left[(n+1) y_{\sigma(i)}-\left(y_{1}+\cdots+y_{n}\right)\right.\right. \\
+\sigma(i)-i-(j-1)] \\
\left.\times(-1)^{n\left(y_{1}+\cdots+y_{n}\right)} q^{\sum_{i=1}^{n}\left[(m n / 2) y_{i}^{2}+(m i-m n / 2-l) y_{i}\right]-m e_{2}\left(y_{1}, \ldots, y_{n}\right)}\right\} \\
=(-1)^{n-1} \prod_{i=1}^{n-1} i ! \prod_{1 \leq r<s \leq n}\left[\left(q^{m}, q^{m} ; q^{m}\right)_{\infty}\right] \prod_{i=1}^{n}\left[\left(q^{m-l}, q^{l}, q^{m} ; q^{m}\right)_{\infty}\right] .
\end{array}
$$

We need to evaluate

$$
\sum_{\sigma \in \mathcal{S}_{n}} \operatorname{sign}(\sigma) \prod_{i=1}^{n-1} \prod_{j=1}^{i}\left[(n+1) y_{\sigma(i)}-\left(y_{1}+\cdots+y_{n}\right)+\sigma(i)-i-(j-1)\right]
$$

First, change the index on the first product so that $i=2, \ldots, n$ instead of $i=1, \ldots, n-$ 1. Now define $\beta(i)=\sigma(i-1)$ for $i=2, \ldots, n$ and $\beta(1)=\sigma(n)$. This means that 
$\operatorname{sign}(\sigma)=(-1)^{n-1} \operatorname{sign}(\beta)$. Thus, the following holds:

$$
\begin{gathered}
\sum_{\sigma \in \mathcal{S}_{n}} \operatorname{sign}(\sigma) \prod_{i=1}^{n-1} \prod_{j=1}^{i}\left[(n+1) y_{\sigma(i)}-\left(y_{1}+\cdots+y_{n}\right)+\sigma(i)-i-(j-1)\right] \\
=(-1)^{n-1} \sum_{\beta \in \mathcal{S}_{n}} \operatorname{sign}(\sigma) \prod_{i=2}^{n} \prod_{j=1}^{i-1}\left[(n+1) y_{\beta(i)}-\left(y_{1}+\cdots+y_{n}\right)\right. \\
+\beta(i)-(i-1)-(j-1)] \\
=(-1)^{n-1} \sum_{\beta \in \mathcal{S}_{n}} \operatorname{sign}(\sigma) \prod_{i=1}^{n} \prod_{j=1}^{i-1}\left[(n+1) y_{\beta(i)}-\left(y_{1}+\cdots+y_{n}\right)\right. \\
\quad+\beta(i)-(i-1)-(j-1)] .
\end{gathered}
$$

Now, the last sum in (4.6) is the same determinant which occurred in Sections 2 and 3. Therefore, we have proven the theorem:

Theorem 4.2. Let $|q|<1$ and let $l$ and $m$ be integers such that $m \geq 2$ and $1 \leq l<m$. Let $n \geq 1$. Then

$$
\begin{aligned}
\left(q^{m} ; q^{m}\right)_{\infty}^{n^{2}}\left(q^{m-l}, q^{l} ; q^{m}\right)_{\infty}^{n} & \\
= & \prod_{i=1}^{n-1} i !^{-1} \sum_{-\infty<y_{1}, \ldots, y_{n}<\infty} \prod_{1 \leq r<s \leq n}\left[(n+1) y_{s}-(n+1) y_{r}+s-r\right] \\
& \quad \times(-1)^{n\left(y_{1}+\cdots+y_{n}\right)} q^{\sum_{i=1}^{n}\left[(m n / 2) y_{i}^{2}+(m i-m n / 2-l) y_{i}\right]-m e_{2}\left(y_{1}, \ldots, y_{n}\right)}
\end{aligned}
$$

where $e_{2}\left(y_{1}, \ldots, y_{n}\right)$ is the second elementary symmetric function of $\left\{y_{1}, \ldots, y_{n}\right\}$.

The case $n=1$ of Theorem 4.2 gives various well-known specializations of the Jacobi triple product identity. We obtain (1.1) when $n=1, m=3$, and $\ell=1$. Taking $m=2$ and $\ell=1$ in (4.7) leads to an expansion for

$$
(q ; q)_{\infty}^{2 n}\left(q^{2} ; q^{2}\right)_{\infty}^{(n-1)^{2}-1}
$$

For example, the case $n=2, m=2$, and $\ell=1$ of (4.7) gives

$$
(q ; q)_{\infty}^{4}=\sum_{-\infty<y_{1}, y_{2}<\infty}\left[1+3\left(y_{2}-y_{1}\right)\right] q^{y_{1}^{2}+y_{2}^{2}+2\left({ }^{1+y_{2}-y_{1}}\right)}
$$

Observe that $\left(y_{1}^{2}+y_{2}^{2}\right)$ and $\left(y_{2}-y_{1}\right)$ are unchanged when $\left(y_{1}, y_{2}\right)$ is reflected through the line $y_{1}+y_{2}=0$, that is when $\left(y_{1}, y_{2}\right) \mapsto\left(-y_{2},-y_{1}\right)$. We then rewrite (4.9) as

$$
\begin{aligned}
(q ; q)_{\infty}^{4}= & \sum_{y_{1}=-\infty}^{\infty}\left(6 y_{1}+1\right) q^{2\left(3 y_{1}^{2}+y_{1}\right)}+\sum_{y_{1}=1}^{\infty} 2 q^{2 y_{1}^{2}} \\
& +2 \sum_{\substack{-\infty<y_{1}, y_{2}<\infty \\
y_{1}+y_{2}>0, y_{1} \neq y_{2}}}\left[1+3\left(y_{2}-y_{1}\right)\right] q^{y_{1}^{2}+y_{2}^{2}+2\left({ }^{1+y_{2}-y_{1}}\right)} .
\end{aligned}
$$

The first sum in (4.10) is a specialized derivative of the quintuple product identity sum, and the second is the classical theta function $\vartheta_{3}\left(0, q^{2}\right)$ minus 1 . By an identity of Ramanujan [2, Entry 8(ix), p. 114], first proven by Gordon [9, (11)], the first 
sum equals $\left(q^{4} ; q^{4}\right)_{\infty}^{3}\left(q^{4} ; q^{8}\right)_{\infty}^{2}$, and a classical identity of Gauss in [1, p. 23, (2.2.12)] implies that the second sum equals $\left[-1+\left(q^{4} ; q^{4}\right)_{\infty}\left(-q^{2} ; q^{4}\right)_{\infty}^{2}\right]$. See $[20$, p. 589] for more detailed discussion of these two sums in (4.10).

The case $n=4, m=2$, and $\ell=1$ of (4.7) gives an expansion for the eighth power of the product side of the Kac-Peterson identity in [13, final equation].

To derive the second family of expansions, we begin with (2.3). Let $m \geq 2$ be an integer and let $l$ be an integer such that $1 \leq l<m$. Replace $q$ by $q^{m}$ in $(2.3)$ and then replace $t$ by $t q^{l}$. We obtain

$$
\begin{aligned}
\left(t q^{l}, t^{-1} q^{m-l} ; q^{m}\right)_{\infty}\left(q^{m} ; q^{m}\right)_{\infty}^{n} \prod_{1 \leq r<s \leq n}\left[\left(1-\frac{x_{r}}{x_{s}}\right)\left(q^{m} \frac{x_{r}}{x_{s}}, q^{m} \frac{x_{s}}{x_{r}} ; q^{m}\right)_{\infty}\right] \\
=\sum_{\substack{-\infty<y_{i}<\infty \\
i=1, \ldots, n}} \sum_{\sigma \in \mathcal{S}_{n}} \operatorname{sign}(\sigma)\left\{\prod_{i=1}^{n}\left[x_{i}^{n y_{\sigma(i)}-\left(y_{1}+\cdots+y_{n}\right)+\sigma(i)-i}\right]\right. \\
\quad \times(-1)^{n\left(y_{1}+\cdots+y_{n}\right)} t^{-\left(y_{1}+\cdots+y_{n}\right)} q^{\left.\sum_{i=1}^{n}\left[\frac{m n}{2} y_{i}^{2}+\left(m i-\frac{m n}{2}-l\right) y_{i}\right]\right\} .}
\end{aligned}
$$

Set $t=1$. Take $\partial^{\left(\begin{array}{c}n \\ 2\end{array}\right)} / \partial x_{1}^{0} \partial x_{2}^{1} \ldots \partial x_{n}^{n-1}$ and then set $x_{1}=\cdots=x_{n}=1$. We have

$$
\begin{aligned}
\prod_{i=1}^{n-1} i ! \cdot\left(q^{l}, q^{m-l} ; q^{m}\right)_{\infty}\left(q^{m} ; q^{m}\right)_{\infty}^{n}\left(q^{m} ; q^{m}\right)_{\infty}^{n(n-1)} \\
=\sum_{\substack{-\infty<y_{i}<\infty \\
i=1, \ldots, n}} \sum_{\sigma \in \mathcal{S}_{n}} \operatorname{sign}(\sigma)\left\{\prod_{i=1}^{n} \prod_{j=1}^{i-1}\left[n y_{\sigma(i)}-\left(y_{1}+\cdots+y_{n}\right)+\sigma(i)-i-(j-1)\right]\right. \\
\quad \times(-1)^{n\left(y_{1}+\cdots+y_{n}\right)} q^{\left.\sum_{i=1}^{n}\left[\frac{m n}{2} y_{i}^{2}+\left(m i-\frac{m n}{2}-l\right) y_{i}\right]\right\} .}
\end{aligned}
$$

Once again we recognize that the inner sum in (4.12) is the same determinant as in (3.5). We have derived the theorem: Theorem 4.3. Let $|q|<1$ and let $l$ and $m$ be integers such that $m \geq 2$ and $1 \leq l<m$.
Let $n \geq 1$. Then

$$
\begin{aligned}
\left(q^{l}, q^{m-l} ; q^{m}\right)_{\infty}\left(q^{m} ; q^{m}\right)_{\infty}^{n^{2}}= & \prod_{i=1}^{n-1} i !^{-1} \sum_{\substack{-\infty<y_{i}<\infty \\
i=1, \ldots, n}} \prod_{1 \leq r<s \leq n}\left[n y_{s}-n y_{r}+s-r\right] \\
& \times(-1)^{n\left(y_{1}+\cdots+y_{n}\right)} q^{\sum_{i=1}^{n}\left[\frac{m n}{2} y_{i}^{2}+\left(m i-\frac{m n}{2}-l\right) y_{i}\right] .}
\end{aligned}
$$

The case $n=1$ of Theorem 4.3 gives various well-known specializations of the Jacobi triple product identity. Note that the cases $n=1$ of the right-hand sides of (4.7) and (4.13) are equal. Thus, the case $n=1, m=3$, and $\ell=1$ of (4.13) also gives (1.1). Now, taking $m=2$ and $\ell=1$ in (4.13) leads to an expansion for

$$
(q ; q)_{\infty}^{2}\left(q^{2} ; q^{2}\right)_{\infty}^{n^{2}-2}
$$

For example, the case $n=2, m=2$, and $\ell=1$ of (4.13) gives

$$
\left[(q ; q)_{\infty}\left(q^{2} ; q^{2}\right)_{\infty}\right]^{2}=\sum_{-\infty<y_{1}, y_{2}<\infty}\left[1+2\left(y_{2}-y_{1}\right)\right] q^{2 y_{1}^{2}+2 y_{2}^{2}+y_{2}-y_{1}}
$$


In the same way that we rewrote (4.9) as (4.10), we find that (4.15) equals

$$
\begin{aligned}
{\left[(q ; q)_{\infty}\left(q^{2} ; q^{2}\right)_{\infty}\right]^{2}=} & \sum_{y_{1}=-\infty}^{\infty}\left(4 y_{1}+1\right) q^{2\left(2 y_{1}^{2}+y_{1}\right)}+\sum_{y_{1}=1}^{\infty} 2 q^{4 y_{1}^{2}} \\
& +2 \sum_{\substack{-\infty<y_{1}, y_{2}<\infty \\
y_{1}+y_{2}>0, y_{1} \neq y_{2}}}\left[1+2\left(y_{2}-y_{1}\right)\right] q^{2 y_{1}^{2}+2 y_{2}^{2}+y_{2}-y_{1}} .
\end{aligned}
$$

The first sum in (4.16) is the case $q \mapsto q^{2}$ of (1.2). Just note that $y_{1} \geq 0$ gives the $k=$ even terms in (1.2) and $y_{1}<0$ gives the odd terms. Thus, the first sum in (4.16) is $\left(q^{2} ; q^{2}\right)_{\infty}^{3}$. The second sum in (4.16) is the classical theta function $\vartheta_{3}\left(0, q^{4}\right)$ minus 1 , and thus equals $\left[-1+\left(q^{8} ; q^{8}\right)_{\infty}\left(-q^{4} ; q^{8}\right)_{\infty}^{2}\right]$. The product in $(4.16)$ is the square of the product side of the Kac-Peterson identity in [13, final equation].

To derive the third family of expansions, we begin with (3.4) and follow the same steps which lead to Theorem 4.3. The only difference is that, in this case, we may allow $0 \leq l<m$ and $m \geq 1$. We obtain the theorem:

Theorem 4.4. Let $|q|<1$ and let $l$ and $m$ be integers such that $m \geq 1$ and $0 \leq l<m$. Let $n \geq 1$. Then

$$
\begin{aligned}
\left(q^{m-l} ; q^{m}\right)_{\infty}\left(q^{m} ; q^{m}\right)_{\infty}^{n^{2}-1} & \\
= & \prod_{i=1}^{n-1} i !^{-1} \sum_{\substack{-\infty<y_{1}, \ldots, y_{n}<\infty \\
y_{1}+\cdots+y_{n} \geq 0}}\left(q^{m} ; q^{m}\right)_{y_{1}+\cdots+y_{n}}^{-1} \prod_{1 \leq r<s \leq n}\left[n y_{s}-n y_{r}+s-r\right] \\
& \quad \times(-1)^{n\left(y_{1}+\cdots+y_{n}\right)} q^{\sum_{i=1}^{n}\left[\frac{m n}{2} y_{i}^{2}+\left(m i-\frac{m n}{2}-l\right) y_{i}\right]}
\end{aligned}
$$

If $m=1$ and $\ell=0$, then we get Theorem 3.1. If $n=1$, we get various specializations of the Euler identity in (3.1). If $m=2$ and $\ell=1$, then (4.17) gives an expansion for

$$
(q ; q)_{\infty}\left(q^{2} ; q^{2}\right)_{\infty}^{n^{2}-2}
$$

For example, the case $n=2, m=2$, and $\ell=1$ of (4.17) gives

$$
(q ; q)_{\infty}\left(q^{2} ; q^{2}\right)_{\infty}^{2}=\sum_{\substack{\infty<y_{1}, y_{2}<\infty \\ y_{1}+y_{2} \geq 0}} \frac{\left[1+2\left(y_{2}-y_{1}\right)\right]}{\left(q^{2} ; q^{2}\right)_{y_{1}+y_{2}}} q^{2 y_{1}^{2}+2 y_{2}^{2}+y_{2}-y_{1}}
$$

where $0<|q|<1$. Since the first sum in $(4.16)$ is $\left(q^{2} ; q^{2}\right)_{\infty}^{3}$, and Slater's identity of Rogers-Ramanujan type from [29, p. 160, eq. 83] can be written as

$$
\sum_{y=0}^{\infty} \frac{q^{2 y^{2}}}{(q ; q)_{2 y}}=\frac{\left(q, q^{7}, q^{8} ; q^{8}\right)_{\infty}\left(q^{6}, q^{10} ; q^{16}\right)_{\infty}}{(q ; q)_{\infty}}
$$

it follows that (4.19) can be transformed into

$$
\begin{aligned}
(q ; q)_{\infty}\left(q^{2} ; q^{2}\right)_{\infty}^{2}=-1 & +\left(q^{2} ; q^{2}\right)_{\infty}^{3}+\frac{\left(q^{2}, q^{14}, q^{16} ; q^{16}\right)_{\infty}\left(q^{12}, q^{20} ; q^{32}\right)_{\infty}}{\left(q^{2} ; q^{2}\right)_{\infty}} \\
& +\sum_{\substack{-\infty<y_{1}, y_{2}<\infty \\
y_{1}+y_{2}>0, y_{1} \neq y_{2}}}\left[1+2\left(y_{2}-y_{1}\right)\right] \frac{q^{2 y_{1}^{2}+2 y_{2}^{2}+y_{2}-y_{1}}}{\left(q^{2} ; q^{2}\right)_{y_{1}+y_{2}}}
\end{aligned}
$$


where $0<|q|<1$. The expression $\left[-1+\left(q^{2} ; q^{2}\right)_{\infty}^{3}\right]$ comes from the $y_{1}+y_{2}=0$, $y_{1} y_{2} \neq 0$ terms in (4.19), and the quotient of products in (4.21) results from applying (4.20) to the single sum of $y_{1}=y_{2} \geq 0$ terms in (4.19).

\section{A proof of the quintuple product identity}

In this section, we give a new derivation of the quintuple product identity [8, Ex. 5.6, p. 134]

$$
\sum_{y=-\infty}^{\infty}(-1)^{y} q^{y(3 y-1) / 2} z^{3 y}\left(1+z q^{y}\right)=(q,-z,-q / z ; q)_{\infty}\left(q z^{2}, q / z^{2} ; q^{2}\right)_{\infty}
$$

where $0<|q|<1$ and $z \neq 0$.

We obtain (5.1) as the case $n=1$ of a $U(n+2)$ extension of the quintuple product identity. We derive this multivariable extension from (4.1) and the Jacobi triple product identity $[8,(1.6 .1)$, p. 12] given by

$$
\left(q,-x q,-x^{-1} ; q\right)_{\infty}=\sum_{y=-\infty}^{\infty} x^{y} q^{\left(y^{2}+y\right) / 2}
$$

where $0<|q|<1$ and $x \neq 0$.

We first use (5.2) to transform the product side of (4.1). Set $x=t x_{i} / x_{n}$ and then take the product of both sides of (5.2) for $i=1, \ldots, n$. This gives

$$
\begin{aligned}
(q)_{\infty}^{n} \prod_{i=1}^{n}\left[\left(-t q \frac{x_{i}}{x_{n}}\right)_{\infty}\right. & \left.\left(-t^{-1} \frac{x_{n}}{x_{i}}\right)_{\infty}\right] \\
& =\sum_{\substack{-\infty<y_{i}<\infty \\
i=1, \ldots, n}} t^{y_{1}+\cdots+y_{n}} x_{n}^{-\left(y_{1}+\cdots+y_{n}\right)} \prod_{i=1}^{n} x_{i}^{y_{i}} \prod_{i=1}^{n} q^{\left(y_{i}^{2}+y_{i}\right) / 2} .
\end{aligned}
$$

Observe that the product side of (5.3) is the second product of (4.1). Thus, we can rewrite (4.1) as

$$
\begin{aligned}
& \sum_{-\infty<y_{1}, \ldots, y_{n}<\infty}\left\{\prod_{1 \leq r<s \leq n}\left[1-\frac{x_{r}}{x_{s}} q^{y_{r}-y_{s}}\right] \prod_{i=1}^{n}\left[\left(x_{i}\right)^{n y_{i}-\left(y_{1}+\cdots+y_{n}\right)}\right]\right. \times(-1)^{(n-1)\left(y_{1}+\cdots+y_{n}\right)} q^{\left[n\left[\left(\frac{y_{1}}{2}\right)+\cdots+\left(\begin{array}{c}
y_{n} \\
2
\end{array}\right)\right]+y_{1}+2 y_{2}+\cdots+n y_{n}\right]} \\
&\left.\times \prod_{i=1}^{n}\left[\left(\frac{x_{i}}{x_{n}}\right)^{y_{i}}\right] q^{-e_{2}\left(y_{1}, \ldots, y_{n}\right)}\right\} t^{y_{1}+\cdots+y_{n}} \\
&=\prod_{1 \leq r<s \leq n}\left[\left(\frac{x_{r}}{x_{s}}\right)_{\infty}\left(q \frac{x_{s}}{x_{r}}\right)_{\infty}\right] \\
& \times \sum_{\substack{-\infty<y_{i}<\infty \\
i=1, \ldots, n}} t^{y_{1}+\cdots+y_{n}} x_{n}^{-\left(y_{1}+\cdots+y_{n}\right)} \prod_{i=1}^{n} x_{i}^{y_{i}} \prod_{i=1}^{n} q^{\left(y_{i}^{2}+y_{i}\right) / 2}
\end{aligned}
$$

where $n \geq 1,0<|q|<1$, and $e_{2}\left(y_{1}, \ldots, y_{n}\right)$ is the second elementary symmetric function of $\left\{y_{1}, \ldots, y_{n}\right\}$. 
Equating the coefficients of $t^{0}$ in (5.4) yields the following identity.

$$
\begin{aligned}
& \sum_{\substack{-\infty<y_{1}, \ldots, y_{n}<\infty \\
y_{1}+\cdots+y_{n}=0}}\left\{\prod_{1 \leq r<s \leq n}\left[1-\frac{x_{r}}{x_{s}} q^{y_{r}-y_{s}}\right] \prod_{i=1}^{n}\left[x_{i}^{(n+1) y_{i}}\right]\right. \\
& \left.\times q^{\frac{n}{2}\left(y_{1}^{2}+\cdots+y_{n}^{2}\right)+y_{2}+2 y_{3}+\cdots+(n-1) y_{n}} q^{-e_{2}\left(y_{1}, \ldots, y_{n}\right)}\right\} \\
& =\prod_{1 \leq r<s \leq n}\left[\left(\frac{x_{r}}{x_{s}}\right)_{\infty}\left(q \frac{x_{s}}{x_{r}}\right)_{\infty}\right] \sum_{\substack{-\infty<y_{1}, \ldots, y_{n}<\infty \\
y_{1}+\cdots+y_{n}=0}} \prod_{i=1}^{n} x_{i}^{y_{i}} \prod_{i=1}^{n} q^{y_{i}^{2} / 2} .
\end{aligned}
$$

If we assume that $n \geq 2$, then we can write $y_{n}=-\left(y_{1}+\cdots+y_{n-1}\right)$. Apply this to (5.5) and simplify. We have

$$
\begin{aligned}
\sum_{-\infty<y_{1}, \ldots, y_{n-1}<\infty} \prod_{1 \leq r<s \leq n-1}\left[1-\frac{x_{r}}{x_{s}} q^{y_{r}-y_{s}}\right] \prod_{i=1}^{n-1}\left[1-\frac{x_{i}}{x_{n}} q^{y_{i}+\left(y_{1}+\cdots+y_{n-1}\right)}\right] \\
\quad \times \prod_{i=1}^{n-1}\left[\left(\frac{x_{i}}{x_{n}}\right)^{(n+1) y_{i}}\right] q^{\sum_{i=1}^{n-1}\left[(n+1) y_{i}^{2}-(n-i) y_{i}\right]+(n+1) e_{2}\left(y_{1}, \ldots, y_{n-1}\right)} \\
=\prod_{1 \leq r<s \leq n}\left[\left(\frac{x_{r}}{x_{s}}\right)_{\infty}\left(q \frac{x_{s}}{x_{r}}\right)_{\infty}\right] \\
\quad \times \sum_{-\infty<y_{1}, \ldots, y_{n-1}<\infty} \prod_{i=1}^{n-1}\left[\left(\frac{x_{i}}{x_{n}}\right)^{y_{i}}\right] q^{y_{1}^{2}+\cdots+y_{n-1}^{2}+e_{2}\left(y_{1}, \ldots, y_{n-1}\right)} .
\end{aligned}
$$

Next, set $x_{i} / x_{n}=z_{i}$ in (5.6), and then rewrite the first product in the right-hand side of (5.6) by means of the identity:

$$
\prod_{1 \leq r<s \leq n}\left[\left(\frac{z_{r}}{z_{s}}\right)_{\infty}\left(q \frac{z_{s}}{z_{r}}\right)_{\infty}\right]=\prod_{1 \leq r<s \leq n-1}\left[\left(\frac{z_{r}}{z_{s}}\right)_{\infty}\left(q \frac{z_{s}}{z_{r}}\right)_{\infty}\right] \prod_{i=1}^{n-1}\left[\left(z_{i}\right)_{\infty}\left(q z_{i}^{-1}\right)_{\infty}\right] .
$$

Letting $n \mapsto n+1$ now yields the theorem:

Theorem 5.1 (A $U(n+2)$ extension of the quintuple product identity). Let $z_{1}, \ldots$, $z_{n}$ be indeterminate. Let $n \geq 1$ and $0<|q|<1$. Then

$$
\begin{aligned}
\sum_{-\infty<y_{1}, \ldots, y_{n}<\infty} & \prod_{1 \leq r<s \leq n}\left[1-\frac{z_{r}}{z_{s}} q^{y_{r}-y_{s}}\right] \prod_{i=1}^{n}\left[1-z_{i} q^{y_{i}+\left(y_{1}+\cdots+y_{n}\right)}\right] \\
\times & \prod_{i=1}^{n}\left[z_{i}^{(n+2) y_{i}}\right] q^{\sum_{i=1}^{n}\left[(n+2) y_{i}^{2}-(n+1-i) y_{i}\right]+(n+2) e_{2}\left(y_{1}, \ldots, y_{n}\right)} \\
= & \prod_{1 \leq r<s \leq n}\left[\left(\frac{z_{r}}{z_{s}}\right)_{\infty}\left(q \frac{z_{s}}{z_{r}}\right)_{\infty}\right] \prod_{i=1}^{n}\left[\left(z_{i}\right)_{\infty}\left(q z_{i}^{-1}\right)_{\infty}\right] \\
& \times \sum_{-\infty<y_{1}, \ldots, y_{n}<\infty} \prod_{i=1}^{n}\left[z_{i}^{y_{i}}\right] q^{y_{1}^{2}+\cdots+y_{n}^{2}+e_{2}\left(y_{1}, \ldots, y_{n}\right)}
\end{aligned}
$$

where $e_{2}\left(y_{1}, \ldots, y_{n}\right)$ is the second elementary symmetric function of $\left\{y_{1}, \ldots, y_{n}\right\}$. 
It is not hard to see that the quintuple product identity in (5.1) is an immediate consequence of (5.2) and the case $n=1$ of (5.8) given by

$$
\sum_{y_{1}=-\infty}^{\infty}\left[\left(1-z_{1} q^{2 y_{1}}\right) z_{1}^{3 y_{1}} q^{3 y_{1}^{2}-y_{1}}\right]=\left(z_{1}\right)_{\infty}\left(q z_{1}^{-1}\right)_{\infty} \sum_{y_{1}=-\infty}^{\infty} z_{1}^{y_{1}} q^{y_{1}^{2}}
$$

In (5.2), take $x \mapsto z_{1} q^{-1 / 2}$ and then set $q \mapsto q^{2}$. Apply the resulting identity to the sum on the right-hand side of (5.9), and then set $z_{1} \mapsto-z$. We obtain

$$
\begin{aligned}
\sum_{y=-\infty}^{\infty}\left[\left(1+z q^{2 y}\right)(-1)^{y} z^{3 y} q^{3 y^{2}-y}\right] & =\left(-z,-q z^{-1} ; q\right)_{\infty}\left(z q, z^{-1} q, q^{2} ; q^{2}\right)_{\infty} \\
& =\left(-z,-z q,-z^{-1} q,-z^{-1} q^{2}, z q, z^{-1} q, q^{2} ; q^{2}\right)_{\infty} \\
& =\left(-z,-z^{-1} q^{2}, q^{2} ; q^{2}\right)_{\infty}\left(z^{2} q^{2}, z^{-2} q^{2} ; q^{4}\right)_{\infty}
\end{aligned}
$$

Finally, let $q \mapsto q^{1 / 2}$. Equation (5.10) then becomes the quintuple product identity in (5.1).

The case $n=1$ of (5.8), via (4.1), is directly related to the $U(3)$ or $A_{2}^{(1)}$ denominator formula. That is, the above derivation of the quintuple product identity in (5.1) followed from three applications of (5.2) (the $A_{1}^{(1)}$ denominator formula), and one of the $A_{2}^{(1)}$ denominator formula. Standard Lie algebra proofs in [17, p. 38] of (5.1) utilized just the $A_{2}^{(2)}$ denominator formula, or in [7, p. 289], the unspecialized character formula for a fundamental standard module for $A_{1}^{(1)}$ (which was equivalent to the denominator formula for $\left.A_{2}^{(2)}\right)$.

\section{A comparison of powers of $\eta$ with those in Macdonald}

In this section, we utilize the classical results of [25, pp. 348-378] and properties of Chebyshev polynomials [6, pp. 183-187], [10, pp. 1032-1033] to derive various formulas and recursions for all the dimensions which yield the same powers of $\eta(q)$ in Theorems 2.3, 3.2, and Macdonald's [18] expansions corresponding to affine root systems of type $A_{\ell}, B_{\ell}, C_{\ell}, B C_{\ell}$, and $D_{\ell}$. These dimensions consist of very sparse "Lucas sequences" [27, pp. 53-74], which we express in terms of Chebyshev polynomials. We also show that the powers $n^{2}-2$ of $(q ; q)_{\infty}$ in Conjecture 3.4, for $n \geq 2$, never appear as a power of $\eta(q)$ in these expansions of Macdonald.

The powers of $\eta(q)$ in Theorems 2.3 and 3.2 are $n^{2}+2$ and $n^{2}$, respectively, for $n \geq 1$. On the other hand, the powers of $\eta(q)$ in Macdonald's expansions corresponding to $A_{\ell}$ $(\ell \geq 1), B_{\ell}(\ell \geq 3), C_{\ell}(\ell \geq 2), B C_{\ell}(\ell \geq 1)$, and $D_{\ell}(\ell \geq 4)$ are $\ell^{2}+2 \ell, 2 \ell^{2}+\ell, 2 \ell^{2}+\ell$, $2 \ell^{2}-\ell$, and $2 \ell^{2}-\ell$, respectively. These expansions can be found in pages $134-135$ $((6)(\mathrm{a})-(\mathrm{b})), 135((6)(\mathrm{a})), 136((6), 137-138((6)(\mathrm{c}))$, and $138(6)$, respectively, of [18].

Except for $\ell=n=1$, in the case of $n^{2}+2=\ell^{2}+2 \ell$, there are no positive integral solutions $(\ell, n)$ to equating $n^{2}+2, n^{2}$, or $n^{2}-2$ with $\ell^{2}+2 \ell$. Similarly, there are no such solutions when equating $n^{2}+2$ with $\ell^{2}$ or $\ell^{2}-2$, or equating $n^{2}$ with $\ell^{2}-2$. We use the analysis in [25, pp. 348-349] and compare $u-v$ and $u+v$ with the factors of $N$ in the Pell equations $u^{2}-v^{2}=N$, for $N=1,2,3$, and 4 .

We now study the rest of the cases in which each of $n^{2}+2, n^{2}, n^{2}+2 n$, or $n^{2}-2$ equals $2 \ell^{2}+\ell$ or $2 \ell^{2}-\ell$. For convenience, we use the variables $x=\ell$ and $y=n$. We 
then seek all positive integral solutions of the following eight quadratic Diophantine equations:

$$
\begin{array}{ll}
2 x^{2}-y^{2}+x-2=0, & 2 x^{2}-y^{2}-x-2=0 \\
2 x^{2}-y^{2}+x=0, & 2 x^{2}-y^{2}-x=0 \\
2 x^{2}-y^{2}+x-2 y=0, & 2 x^{2}-y^{2}-x-2 y=0 \\
2 x^{2}-y^{2}+x+2=0, & 2 x^{2}-y^{2}-x+2=0 .
\end{array}
$$

Completing the square in (6.1)-(6.4) as in [25, p. 375] immediately gives the following Pell equations:

$$
\begin{array}{ll}
u^{2}-8 v^{2}=-136 & \text { where } x=\frac{1}{4}(v-1) \text { and } y=\frac{1}{8} u, \\
u^{2}-8 v^{2}=-136 & \text { where } x=\frac{1}{4}(v+1) \text { and } y=\frac{1}{8} u, \\
u^{2}-8 v^{2}=-8 & \text { where } x=\frac{1}{4}(v-1) \text { and } y=\frac{1}{8} u, \\
u^{2}-8 v^{2}=-8 & \text { where } x=\frac{1}{4}(v+1) \text { and } y=\frac{1}{8} u, \\
u^{2}-8 v^{2}=56 & \text { where } x=\frac{1}{4}(v-1) \text { and } y=\frac{1}{8} u-1, \\
u^{2}-8 v^{2}=56 & \text { where } x=\frac{1}{4}(v+1) \text { and } y=\frac{1}{8} u-1, \\
u^{2}-8 v^{2}=120 & \text { where } x=\frac{1}{4}(v-1) \text { and } y=\frac{1}{8} u, \\
u^{2}-8 v^{2}=120 & \text { where } x=\frac{1}{4}(v+1) \text { and } y=\frac{1}{8} u .
\end{array}
$$

At this point, it is useful to recall the Chebyshev polynomials $T_{n}(x)$ and $U_{n}(x)$ defined in $[6, p .184]$ by

$$
\begin{array}{ll}
T_{n}(\cos \theta):=\cos n \theta & \text { for } \quad n=0,1,2, \ldots, \\
U_{n}(\cos \theta):=\frac{\sin (n+1) \theta}{\sin \theta} & \text { for } \quad n=-1,0,1,2, \ldots, \text { where } x=\cos \theta .
\end{array}
$$

Note that we have the three-term recursions from $[6$, p. 185, (16)] given by

$$
\begin{aligned}
& T_{n}(x)=2 x T_{n-1}(x)-T_{n-2}(x), \text { with }\left(T_{0}(x), T_{1}(x)\right)=(1, x), \text { for } n \geq 2, \\
& U_{n}(x)=2 x U_{n-1}(x)-U_{n-2}(x), \text { with }\left(U_{-1}(x), U_{0}(x)\right)=(0,1), \text { for } n \geq 1 .
\end{aligned}
$$

Thus, if $x$ is an integer, then so are $T_{n}(x)$ and $U_{n}(x)$. Our use of Chebyshev polynomials is motivated by [25, p. 389 , Prob. 35].

In order to state our first theorem involving (6.7) and (6.8), which compares $n^{2}$ with $2 \ell^{2} \pm \ell$, we also need the Pell numbers $\left\{P_{r}\right\}$ from [27, pp. 55, 72], [30, sequence M1413] determined by

$$
P_{r}=2 P_{r-1}+P_{r-2}, \quad \text { with } \quad\left(P_{0}, P_{1}\right)=(0,1), \quad \text { for } \quad r \geq 2 .
$$

The $\left\{P_{2 r+2}\right\}$ are the bisection of Pell numbers in [30, sequence M2030] and $\left\{P_{2 r+1}\right\}$ are the classical Pythagorean triangles sequence in [28, pp. 16-20], [30, sequence M3955].

We now have the theorem: 
Theorem 6.1. All positive integral pairs $(\ell, n)$ for which Theorem 3.2 and Macdonald's $B_{\ell}, C_{\ell}, B C_{\ell}$, and $D_{\ell}$ results give expansions for the same power $n^{2}$, as respectively, $2 \ell^{2}+\ell$ or $2 \ell^{2}-\ell$, of $\eta(q)$ are given by $(\ell, n)=\left(x_{r}, y_{r}\right)$, with

$$
\begin{array}{ll}
\left(x_{r}, y_{r}\right)=\left(P_{2 r+2}^{2}, \frac{1}{2} P_{4 r+4}\right)=\left(4 U_{r}^{2}(3), U_{2 r+1}(3)\right) & \text { for } r \geq 0 \\
\left(x_{r}, y_{r}\right)=\left(P_{2 r+1}^{2}, \frac{1}{2} P_{4 r+2}\right)=\left(\left(U_{r}(3)-U_{r-1}(3)\right)^{2}, U_{2 r}(3)\right) & \text { for } r \geq 0,
\end{array}
$$

respectively, where $P_{r}$ are the Pell numbers in (6.17), and $T_{r}(x)$ and $U_{r}(x)$ are the Chebyshev polynomials in (6.15) and (6.16). The $P_{2 r+1}=U_{r}(3)-U_{r-1}(3)$ in (6.19) is the hypotenuse of the $r$-th Pythagorean triangle with consecutive integral legs.

The $y_{r}$ in (6.18) and (6.19) are determined by the recursion

$$
y_{r}=34 y_{r-1}-y_{r-2} \quad \text { for } \quad r \geq 2
$$

where $\left(y_{0}, y_{1}\right)$ equals $(6,204)$ or $(1,35)$, respectively. The $x_{r}$ in $(6.18)$ are determined by

$$
x_{r}=34 x_{r-1}-x_{r-2}+8 \quad \text { for } r \geq 2
$$

where $\left(x_{0}, x_{1}\right)=(4,144)$, and the $x_{r}$ in (6.19) are determined by

$$
x_{r}=34 x_{r-1}-x_{r-2}-8 \text { for } r \geq 2
$$

where $\left(x_{0}, x_{1}\right)=(1,25)$. Except for $\left(x_{0}, y_{0}\right)=(1,1)$, we have $x_{r}<y_{r}$ in $(6.18)$ and (6.19).

Substituting (6.18) and (6.19) into (6.2) yields interesting identities for the Pell numbers $P_{r}$ and specialized Chebyshev polynomials. Additional relationships for the $\left\{x_{r}\right\}$ and $\left\{y_{r}\right\}$ in (6.18) and (6.19) follow from the material in [26], [27, pp. 53-74], [6, pp. 183-187], and [10, pp. 1032-1033].

We are led to an elegant generalization of Theorem 6.1 by considering the four families of quadratic Diophantine quations in $x$ and $y$ determined by:

$$
\begin{aligned}
& u^{2}-\left(m^{2}-1\right) v^{2}=-\left(m^{2}-1\right) \quad \text { where } \quad x=\frac{v-1}{m+1} \quad \text { and } \quad y=\frac{u}{m^{2}-1} \\
& u^{2}-\left(m^{2}-1\right) v^{2}=-\left(m^{2}-1\right) \quad \text { where } \quad x=\frac{v+1}{m+1} \quad \text { and } \quad y=\frac{u}{m^{2}-1} \\
& u^{2}-\left(m^{2}-1\right) v^{2}=-\left(m^{2}-1\right) \quad \text { where } \quad x=\frac{v-1}{m^{2}-1} \quad \text { and } \quad y=\frac{u}{m^{2}-1} \\
& u^{2}-\left(m^{2}-1\right) v^{2}=-\left(m^{2}-1\right) \quad \text { where } \quad x=\frac{v-m}{m^{2}-1} \quad \text { and } \quad y=\frac{u}{m^{2}-1}
\end{aligned}
$$

where $m=2,3,4, \ldots$.

From the case $D=m^{2}-1, u=m-1$, and $v=1$ of Corollary 6.35.1 in [25, p. 354], we have that $(u, v)=(m, 1)$ is the fundamental solution of $u^{2}-\left(m^{2}-1\right) v^{2}=1$. Furthermore, this fact and Theorem 6.43 in [25, p. 363] imply that any fundamental solution $(u, v)$ of

$$
u^{2}-\left(m^{2}-1\right) v^{2}=-\left(m^{2}-1\right)
$$

must satisfy the inequalities

$$
0 \leq v \leq \sqrt{(m+1) / 2} \quad \text { and } \quad 0 \leq|u| \leq(m-1) \sqrt{(m+1) / 2}
$$


Checking $v$ and solving for $u$, we find that $(u, v)=(0,1)$ is one such fundamental solution. For $m=3,(0,1)$ is the only fundamental solution of (6.27). Keeping in mind (6.7) and (6.8), it is not hard to see that Theorem 6.1 is a consequence of the case $m=3$ of the following theorem.

Theorem 6.2. Let $m \geq 2$. All positive integral pairs of solutions $\left(x_{r}, y_{r}\right)$ to (6.23)(6.26) corresponding to the fundamental solution $(u, v)=(0,1)$ of $(6.27)$ are given by:

$$
\begin{aligned}
& \left(x_{r}, y_{r}\right)=\left(\frac{T_{2 r+2}(m)-1}{m+1}, U_{2 r+1}(m)\right)=\left(2(m-1) U_{r}^{2}(m), U_{2 r+1}(m)\right) \text { for } r \geq 0 \\
& \left(x_{r}, y_{r}\right)=\left(\frac{T_{2 r+1}(m)+1}{m+1}, U_{2 r}(m)\right)=\left(\left(U_{r}(m)-U_{r-1}(m)\right)^{2}, U_{2 r}(m)\right) \text { for } r \geq 0 \\
& \left(x_{r}, y_{r}\right)=\left(\frac{T_{2 r+2}(m)-1}{m^{2}-1}, U_{2 r+1}(m)\right)=\left(2 U_{r}^{2}(m), U_{2 r+1}(m)\right) \text { for } r \geq 0, \\
& \left(x_{r}, y_{r}\right)=\left(\frac{T_{2 r+3}(m)-m}{m^{2}-1}, U_{2 r+2}(m)\right)=\left(2 U_{r}(m) U_{r+1}(m), U_{2 r+2}(m)\right) \text { for } r \geq 0
\end{aligned}
$$

respectively, where $T_{r}(x)$ and $U_{r}(x)$ are the Chebyshev polynomials in (6.15) and (6.16).

The $y_{r}$ in (6.29)-(6.32) are determined by the recursion

$$
y_{r}=2\left(2 m^{2}-1\right) y_{r-1}-y_{r-2} \quad \text { for } \quad r \geq 2
$$

where $\left(y_{0}, y_{1}\right)$ equals $\left(2 m, U_{3}(m)\right),\left(1, U_{2}(m)\right),\left(2 m, U_{3}(m)\right)$, and $\left(4 m^{2}-1, U_{4}(m)\right)$, respectively. The $x_{r}$ are determined by the recursions

$$
x_{r}=2\left(2 m^{2}-1\right) x_{r-1}-x_{r-2}+c(m) \quad \text { for } r \geq 2,
$$

with $c(m)$ given by

$$
4(m-1), \quad-4(m-1), \quad 4, \quad \text { and } 4 m,
$$

respectively, and where $\left(x_{0}, x_{1}\right)$ equals $\left(2(m-1), 8(m-1) m^{2}\right),\left(1,(2 m-1)^{2}\right),\left(2,8 m^{2}\right)$, and $\left(4 m, 4 m\left(4 m^{2}-1\right)\right)$, respectively.

Define $P_{r}(m)$ by $P_{2 r+2}(m):=\sqrt{2(m-1)} U_{r}(m)$ and $P_{2 r+1}(m):=U_{r}(m)-U_{r-1}(m)$, with $P_{0}(m):=0$ and $U_{r}(x)$ determined by (6.16) for $r \geq 0$. We then have

$$
P_{r}(m)=\sqrt{2(m-1)} P_{r-1}(m)+P_{r-2}(m) \quad \text { for } \quad r \geq 2
$$

where $\left(P_{0}, P_{1}\right)=(0,1)$.

Before proving Theorem 6.2, we need the following identities from [6, pp. 183-187], and [10, pp. 1032-1033] for Chebyshev polynomials.

First, a slight rewriting of $[10$, p. $1032,8.940$ (1) and (2)] gives

$$
\begin{aligned}
& T_{r}(x)=\frac{1}{2}\left[\left(x+\sqrt{x^{2}-1}\right)^{r}+\left(x-\sqrt{x^{2}-1}\right)^{r}\right] \\
& U_{r}(x)=\frac{1}{2 \sqrt{x^{2}-1}}\left[\left(x+\sqrt{x^{2}-1}\right)^{r+1}-\left(x-\sqrt{x^{2}-1}\right)^{r+1}\right],
\end{aligned}
$$


for $r \geq 0$. Next, from [6, p. 187, (34)-corrected, and (36)], we have

$$
\begin{aligned}
& T_{n+m}(x)+T_{n-m}(x)=2 T_{m}(x) T_{n}(x), \quad \text { with } n \geq m, \\
& U_{n+m-1}(x)+U_{n-m-1}(x)=2 T_{m}(x) U_{n-1}(x), \quad \text { with } n>m \text {. }
\end{aligned}
$$

Equations (3) and (4) from [6, p. 184] are

$$
T_{n}(x)=U_{n}(x)-x U_{n-1}(x) \text { and }\left(1-x^{2}\right) U_{n-1}(x)=x T_{n}(x)-T_{n+1}(x) .
$$

Finally, we have:

$$
\begin{aligned}
& T_{2 r+2}(x)-1=2\left(x^{2}-1\right) U_{r}^{2}(x), \\
& T_{2 r+1}(x)+1=(x+1)\left(U_{r}(x)-U_{r-1}(x)\right)^{2}, \\
& T_{2 r+1}(x)-x=2\left(x^{2}-1\right) U_{r-1}(x) U_{r}(x),
\end{aligned}
$$

for $r \geq 0$. Equation (6.42) is the case $n=m=r+1$ of $[6$, p. 187, (35)] and is the corrected version of $[6$, p. 187, (39)]. Equation (6.44) is the case $n=r+1, m=r$ of $[6, \mathrm{p} .187,(35)]$. Use the first relation in (6.41), followed by $(6.37)$ and $(6.38)$ to prove (6.43).

We now prove Theorem 6.2. Recall that $(u, v)=(m, 1)$ is the fundamental solution of $u^{2}-\left(m^{2}-1\right) v^{2}=1$. Thus, by Theorem 6.44 of [25, p. 364], it follows that all positive integral solutions $\left(u_{r}, v_{r}\right)$ of (6.27) of the class $K$ of any given fundamental solution $\left(u_{0}, v_{0}\right)$ are determined by the coupled recursions

$$
u_{r}=m u_{r-1}+\left(m^{2}-1\right) v_{r-1} \text { and } v_{r}=u_{r-1}+m v_{r-1} \text { for } r \geq 1 \text {, }
$$

with $m \geq 2$. Using standard matrix methods which utilize a Jordan decomposition combined with (6.37) and (6.38), we find that

$$
\begin{array}{ll}
u_{r}=u_{0} T_{r}(m)+v_{0}\left(m^{2}-1\right) U_{r-1}(m) & \text { for } r \geq 0, \\
v_{r}=u_{0} U_{r-1}(m)+v_{0} T_{r}(m) & \text { for } r \geq 0 .
\end{array}
$$

Taking $\left(u_{0}, v_{0}\right)=(0,1)=\left(U_{-1}(m), T_{0}(m)\right)$, we obtain

$$
u_{r}=\left(m^{2}-1\right) U_{r-1}(m) \text { and } v_{r}=T_{r}(m) \text { for } r \geq 0 .
$$

Equation (6.48) also follows by observing that (6.41) implies that (6.48) satisfies (6.45) with $\left(u_{0}, v_{0}\right)=(0,1)$.

Applying the case $m \geq 2$ of $(6.45)$ to $\left(u_{r}, v_{r}\right)$ interchanges the conditions

$$
u \equiv 0 \quad\left(\bmod m^{2}-1\right) \quad \text { and } \quad v \equiv 1 \quad(\bmod m+1),
$$

with

$$
u \equiv 0 \quad\left(\bmod m^{2}-1\right) \quad \text { and } \quad v \equiv-1 \quad(\bmod m+1)
$$

and also

$$
u \equiv 0 \quad\left(\bmod m^{2}-1\right) \quad \text { and } \quad v \equiv 1 \quad\left(\bmod m^{2}-1\right)
$$

with

$$
u \equiv 0 \quad\left(\bmod m^{2}-1\right) \quad \text { and } \quad v \equiv m \quad\left(\bmod m^{2}-1\right) .
$$

It now follows that (6.42)-(6.44) and the conditions (6.49) and (6.50) applied to (6.48) and (6.23)-(6.26) yield (6.29)-(6.32).

The recursions in (6.33) and (6.34) are consequences of (6.39), (6.40), and the leftmost equalities in (6.29)-(6.32). If $y_{r}$ equals any of $U_{2 r+1}(x), U_{2 r}(x)$, or $U_{2 r+2}(x)$, then $y_{r}$ satisfies (6.33) with $m=x$. Just consider $(6.40)$ with $(m, n)$ equal to $(2,2 r)$, 
$(2,2 r-1)$, or $(2,2 r+1)$, respectively, and note that $T_{2}(x)=\left(2 x^{2}-1\right)$. If $b$ is any constant independent of $r$, then each of $b T_{2 r+2}(x), b T_{2 r+1}(x)$, and $b T_{2 r+3}(x)$ also satisfy (6.33) with $m=x$. This time, look at $(6.39)$ with $(m, n)$ equal to $(2,2 r),(2,2 r-1)$, or $(2,2 r+1)$, respectively. To recover the recursions for $x_{r}$ in (6.34), observe that if $z_{r}$ satisfies (6.33) and $x_{r}=z_{r}+e$, then $x_{r}$ satisfies $x_{r}=$ $2\left(2 m^{2}-1\right) x_{r-1}-x_{r-2}+4 e\left(1-m^{2}\right)$.

The case $r \mapsto 2 r+1$ of (6.36) follows directly from (6.16), while the case $r \mapsto 2 r+2$ is immediate from the definition of the $P_{r}(m)$.

This completes the proof of Theorem 6.2.

The same analysis responsible for Theorem 6.2 specializes to yield the positive integral solutions $\left(x_{r}, y_{r}\right)$ to (6.1), (6.3), and (6.4) via (6.5), (6.6), and (6.9)-(6.12).

We have the theorem:

Theorem 6.3. Let $T_{r}(x)$ and $U_{r}(x)$ be the Chebyshev polynomials in (6.15) and (6.16). Then, all positive integral pairs of solutions $\left(x_{r}, y_{r}\right)$ to (6.5) are given by:

$$
\begin{aligned}
\left(x_{r}, y_{r}\right) & =\left(2 U_{2 r-1}(3)+\frac{5}{4} T_{2 r}(3)-\frac{1}{4}, T_{2 r}(3)+5 U_{2 r-1}(3)\right) \\
& =\left(2 U_{2 r-1}(3)+T_{2 r}(3)+4 U_{r-1}^{2}(3), T_{2 r}(3)+5 U_{2 r-1}(3)\right) \quad \text { for } r \geq 0,
\end{aligned}
$$

and

$$
\begin{aligned}
\left(x_{r}, y_{r}\right) & =\left(-2 U_{2 r+1}(3)+\frac{5}{4} T_{2 r+2}(3)-\frac{1}{4},-T_{2 r+2}(3)+5 U_{2 r+1}(3)\right) \\
& =\left(-2 U_{2 r+1}(3)+T_{2 r+2}(3)+4 U_{r}^{2}(3),-T_{2 r+2}(3)+5 U_{2 r+1}(3)\right) \quad \text { for } r \geq 0
\end{aligned}
$$

All positive integral pairs of solutions $\left(x_{r}, y_{r}\right)$ to (6.6) are given by

$$
\begin{aligned}
\left(x_{r}, y_{r}\right) & =\left(2 U_{2 r}(3)+\frac{5}{4} T_{2 r+1}(3)+\frac{1}{4}, T_{2 r+1}(3)+5 U_{2 r}(3)\right) \\
& =\left(2 U_{2 r}(3)+T_{2 r+1}(3)+\left(U_{r}(3)-U_{r-1}(3)\right)^{2}, T_{2 r+1}(3)+5 U_{2 r}(3)\right) \text { for } r \geq 0
\end{aligned}
$$

and

$$
\begin{aligned}
&\left(x_{r}, y_{r}\right)=\left(-2 U_{2 r}(3)+\frac{5}{4} T_{2 r+1}(3)+\frac{1}{4},-T_{2 r+1}(3)+5 U_{2 r}(3)\right) \\
&=\left(-2 U_{2 r}(3)+T_{2 r+1}(3)+\left(U_{r}(3)-U_{r-1}(3)\right)^{2},-T_{2 r+1}(3)+5 U_{2 r}(3)\right) \\
& \text { for } r \geq 0 . \quad
\end{aligned}
$$

All positive integral pairs of solutions $\left(x_{r}, y_{r}\right)$ to (6.9) are given by

$$
\begin{aligned}
\left(x_{r}, y_{r}\right) & =\left(2 U_{2 r+1}(3)+\frac{1}{4} T_{2 r+2}(3)-\frac{1}{4}, T_{2 r+2}(3)+U_{2 r+1}(3)-1\right) \\
& =\left(2 U_{2 r+1}(3)+4 U_{r}^{2}(3), T_{2 r+2}(3)+U_{2 r+1}(3)-1\right) \quad \text { for } r \geq 0
\end{aligned}
$$

and

$$
\begin{aligned}
\left(x_{r}, y_{r}\right) & =\left(2 U_{2 r}(3)-\frac{1}{4} T_{2 r+1}(3)-\frac{1}{4}, T_{2 r+1}(3)-U_{2 r}(3)-1\right) \\
& =\left(2 U_{2 r}(3)-\left(U_{r}(3)-U_{r-1}(3)\right)^{2}, T_{2 r+1}(3)-U_{2 r}(3)-1\right) \quad \text { for } r \geq 0 .
\end{aligned}
$$


All positive integral pairs of solutions $\left(x_{r}, y_{r}\right)$ to $(6.10)$ are given by

$$
\begin{aligned}
\left(x_{r}, y_{r}\right) & =\left(2 U_{2 r+1}(3)-\frac{1}{4} T_{2 r+2}(3)+\frac{1}{4}, T_{2 r+2}(3)-U_{2 r+1}(3)-1\right) \\
& =\left(2 U_{2 r+1}(3)-4 U_{r}^{2}(3), T_{2 r+2}(3)-U_{2 r+1}(3)-1\right) \quad \text { for } r \geq 0
\end{aligned}
$$

and

$$
\begin{aligned}
\left(x_{r}, y_{r}\right) & =\left(2 U_{2 r}(3)+\frac{1}{4} T_{2 r+1}(3)+\frac{1}{4}, T_{2 r+1}(3)+U_{2 r}(3)-1\right) \\
& =\left(2 U_{2 r}(3)+\left(U_{r}(3)-U_{r-1}(3)\right)^{2}, T_{2 r+1}(3)+U_{2 r}(3)-1\right) \quad \text { for } r \geq 0 .
\end{aligned}
$$

There are no positive integral pairs of solutions $\left(x_{r}, y_{r}\right)$ to either (6.11) or (6.12).

The $x_{r}$ in (6.51), (6.52), (6.55), and (6.56) are determined by (6.21) where $\left(x_{0}, x_{1}\right)$ equals $(1,33),(9,313),(16,552)$, or $(1,45)$, respectively. The $x_{r}$ in $(6.53),(6.54)$, $(6.57)$, and (6.58) are determined by $(6.22)$ where $\left(x_{0}, x_{1}\right)$ equals $(6,194),(2,54)$, $(8,264)$, or $(3,95)$, respectively. The $y_{r}$ in $(6.51)-(6.54)$ are determined by $(6.20)$ where $\left(y_{0}, y_{1}\right)$ equals $(1,47),(13,443),(8,274)$, or $(2,76)$, respectively. The $y_{r}$ in (6.55)-(6.58) are determined by

$$
y_{r}=34 y_{r-1}-y_{r-2}+32 \quad \text { for } \quad r \geq 2
$$

where $\left(y_{0}, y_{1}\right)$ equals $(22,780),(1,63),(10,372)$, or $(3,133)$, respectively. Except where $\left(x_{0}, y_{0}\right)$ equals $(1,1),(2,2),(1,1)$, or $(3,3)$ in $(6.51),(6.54),(6.56)$, or $(6.58)$, we have $x_{r}<y_{r}$.

The proof of Theorem 6.3 depends upon first finding the fundamental solutions $(u, v)$ of $u^{2}-8 v^{2}=N$ for $N=-136,56$, and 120. For $N=-136$, we have from Theorem 6.43 of $[25$, p. 363] that any fundamental solution must satisfy $0 \leq v \leq 5$ and $0 \leq|u| \leq 11$. This yields all such fundamental solutions $(u, v)=(8,5)$ and $(u, v)=(-8,5)$. On the other hand, for $N=56$ or $N=120$, we have from Theorem 6.42 of [25, pp. 362-363] that any fundamental solution must satisfy $0 \leq v \leq 2$ and $0 \leq|u| \leq 10$, or $0 \leq v \leq 3$ and $0 \leq|u| \leq 15$, respectively. For the $N=56$ case, we find all such fundamental solutions $(u, v)=(8,1)$ and $(u, v)=(-8,1)$, in which we use $(u, v)=(8,-1)$ instead of $(u, v)=(-8,1)$. For the case $N=120$, there are no such fundamental solutions. Thus, there are no positive integral pairs of solutions $\left(x_{r}, y_{r}\right)$ to either (6.11) or (6.12). Having obtained the fundamental solutions, the formulas in (6.51)-(6.58) are consequences of the case $m=3$ of $(6.42),(6.43),(6.45)-(6.47)$, and (6.49).

From the analysis in the proof of Theorem 6.2, we have that if $y_{r}$ is any of $U_{2 r}(x)$, $U_{2 r+1}(x), T_{2 r}(x), T_{2 r+1}(x)$, or any linear combination of these, then $y_{r}$ satisfies (6.33) with $m=x$. Corresponding recursions for $y_{r}+e$, with $e$ a constant, are immediate. Specializing these remarks to $x=3$ yields all the recursions in Theorem 6.3. Another motivation for our recursions is provided by comparing the well-known formula in $[5$, p. $91,(3.7 .2)]$ or $[27$, pp. $54-55]$ with $(6.37)-(6.38)$ and $(6.46)-(6.47)$.

Theorem 6.3 immediately implies the consequences:

Corollary 6.4. All positive integral pairs $(\ell, n)$ for which Theorem 2.3 and Macdonald's $B_{\ell}, C_{\ell}, B C_{\ell}$, and $D_{\ell}$ results give expansions for the same power $n^{2}+2$, as respectively $2 \ell^{2}+\ell$ or $2 \ell^{2}-\ell$, of $\eta(q)$ are given by $(\ell, n)=\left(x_{r}, y_{r}\right)$, with $\left(x_{r}, y_{r}\right)$ in (6.51) and (6.52), or (6.53) and (6.54), respectively. 
There are no positive integral pairs $(\ell, n)$ for which Conjecture 3.4 and Macdonald's $B_{\ell}, C_{\ell}, B C_{\ell}$, and $D_{\ell}$ results give expansions for the same power $n^{2}-2$ of $(q ; q)_{\infty}$, as respectively $2 \ell^{2}+\ell$ or $2 \ell^{2}-\ell$, of $\eta(q)$.

Acknowledgments. S.C. Milne was partially supported by National Security Agency grant MDA 904-97-1-0019.

\section{References}

1. G. E. Andrews, The Theory of Partitions, Encyclopedia of Mathematics and Its Applications (G.C. Rota, ed.), vol. 2, Addison-Wesley, Reading, Mass., 1976; reissued by Cambridge University Press, Cambridge, 1985.

2. B. Berndt, Ramanujan's Notebooks, Part III, Springer-Verlag, New York, 1991.

3. S. Bhargava, A simple proof of the quintuple product identity, J. Indian Math Soc. 61 (1995), 226-228.

4. S. Bhargava and D. Somashekara, Some eta-function identities deducible from Ramanujan's ${ }_{1} \psi_{1}$ summation, J. Math. Anal. Appl. 176 (1993), 554-560.

5. J. M. and P. B. Borwein, Pi and the AGM, John Wiley \& Sons, Inc., New York, 1987.

6. A. Erdélyi, A. Magnus, F. Oberhettinger, and F. Tricomi, Higher Transcendental Functions, Bateman Manuscript Project (A. Erdélyi, ed.), vol. II, McGraw-Hill Book Co., New York, 1953; reissued by Robert E. Krieger Pub. Co., Malabar, Florida, 1981, 1985.

7. A. Feingold and J. Lepowsky, The Weyl-Kac character formula and power series identities, Adv. in Math. 29 (1978), 271-309.

8. G. Gasper and M. Rahman, Basic Hypergeometric Series, Encyclopedia of Mathematics and Its Applications (G.-C. Rota, ed.), vol. 35, Cambridge University Press, Cambridge, 1990.

9. B. Gordon, Some identities in combinatorial analysis, Quart. J. Math. Oxford Ser. 212 (1961), 285-290.

10. I. S. Gradshteyn and I. M. Ryzhik, Table of Integrals, Series, and Products, 4th ed., Academic Press, San Diego, 1980; Translated from the Russian by Scripta Technica, Inc., and edited by A. Jeffrey.

11. G. H. Hardy and E. M. Wright, An Introduction to the Theory of Numbers, 5th ed., Oxford University Press, Oxford, 1979.

12. C. G. J. Jacobi, Fundamenta Nova Theoriae Functionum Ellipticarum, Regiomonti. Sumptibus fratrum Bornträger, 1829; reprinted in Jacobi's Gesammelte Werke, vol 1, Reimer, Berlin, 18811891, pp. 49-239; reprinted by Chelsea, New York, 1969; now distributed by The American Mathematical Society, Providence, RI.

13. V. G. Kac and D. H. Peterson, Affine Lie algebras and Hecke modular forms, Bull. Amer. Math. Soc. (N.S.) 3 (1980), 1057-1061.

14. G. Köhler, Some eta-identities arising from theta series, Math. Scand. 66 (1990), 147-154.

15. V. Leininger and S. Milne, Expansions for $(q)_{\infty}^{n^{2}+2 n}$ and basic hypergeometric series in $U(n)$, Discrete Math., in press.

16. J. Lepowsky, Macdonald-type identities, Adv. in Math. 27 (1978), 230-234.

17. J. Lepowsky and S. Milne, Lie algebraic approaches to classical partition identities, Adv. in Math. 29 (1978), 15-59.

18. I. G. Macdonald, Affine root systems and Dedekind's $\eta$-function, Invent. Math. 15 (1972), 91143.

19. S. C. Milne, An elementary proof of the Macdonald identities for $A_{\ell}^{(1)}$, Adv. in Math. 57 (1985), 34-70.

20. — The $C_{\ell}$ Rogers-Selberg Identity, SIAM J. Math. Anal. 25 (1994), 571-595.

21. _ Balanced ${ }_{3} \phi_{2}$ summation theorems for $U(n)$ basic hypergeometric series, Adv. in Math. 131 (1997), 93-187.

22. —_ A new $U(n)$ generalization of the Jacobi triple product identity, preprint.

23. T. Muir and W. H. Metzler, A Treatise of the Theory of Determinants, Longmans, Green, and Co., New York, 1933.

24. S. Ramanujan, On certain arithmetical functions, Trans. Cambridge Philos. Soc. 22 (1916), 159184; reprinted in Collected Papers of Srinivasa Ramanujan, Chelsea, New York, 1962, pp. 136-162.

25. D. Redmond, Number Theory: An Introduction, Marcel Dekker, New York, 1996. 
26. P. Ribenboim, The Fibonacci numbers and the Arctic Ocean. In: Proceedings of the Second Gaussian Symposium, München, 1994 (M. Behara, R. Fritsch, R. G. Lintz, eds.), Symposia Gaussian, Conf. A, W. de Gruyter, Berlin, 1995, pp.41-83.

27. ㄴ. The New Book of Prime Number Records, 3rd ed., Springer-Verlag, New York, 1996.

28. W. Sierpinski, Pythagorean Triangles, The Scripta Mathematica Studies, vol. 9, Graduate School of Science, Yeshiva University, New York, 1962; Translated from the Polish by Dr. Ambikeshwar Sharma.

29. L. J. Slater, Further identities of the Rogers-Ramanujan type, Proc. London Math. Soc. (2) 54 (1952), 147-167.

30. N. J. A. Sloane and Simon Plouffe, The Encyclopedia of Integer Sequences, Academic Press, San Diego, 1995.

31. M. A. Stern, Ueber einen Satz aus der Determinantentheorie, J. Reine Angew. Math. 66 (1865), 285-288.

32. N. Sthanumoorthy and M. Tamba, $A$ note on a generalization of Macdonald's identities for $A_{\ell}$ and $B_{\ell}$, Proc. Indian Acad. Sci. Math. Sci. 104 (1994), 377-383.

33. S. Wolfram, The Mathematica Book, 3rd ed., Wolfram Media/Cambridge University Press, Cambridge, 1996.

The University of Akron, Akron, OH 44325

E-mail: vleining@bridgewater.edu

Department of Mathematics, The Ohio State University, Columbus, OH 43210

E-mail: milne@math.ohio-state.edu 